\title{
Evolution of Comets Into Asteroids
}

\author{
Paul R. Weissman \\ Jet Propulsion Laboratory \\ William F. Bottke Jr. and Harold F. Levison \\ Southwest Research Institute
}

\begin{abstract}
In the past decade, new observations and new research tools have afforded us a better understanding of the interrelationships between comets and asteroids. The extensive automated surveys for near-Earth objects (NEOs) have serendipitously discovered many objects in cometlike orbits. Dynamical simulation codes have improved to where we can simulate the detailed orbital behavior of large swarms of test particles as they move out from various storage reservoirs, such as the Oort cloud and the Kuiper belt. Physical studies of both comets and asteroids have given us a far better understanding of the nature of these bodies and have identified possible discriminators to be used in comparing them. As a result, we can now identify likely dormant or extinct comet candidates among the asteroid population. It appears that $\sim 6 \% \pm 4 \%$ (or perhaps more) of the NEO population is derived from Jupiter-family comets. Also, it is highly likely that many asteroids in eccentric orbits with large semi-major axes and large inclinations are derived from the Oort cloud. However, we must also recognize that some small fraction of the Oort cloud population is likely to consist of asteroidal bodies ejected there during the clearing of the planetary zones in the early solar system. Additional physical and dynamical studies are required to continue to improve our knowledge of the interrelationships between comets and asteroids, and to help identify likely extinct comet candidates.
\end{abstract}

\section{Introduction}

Solar system astronomers have long speculated on the possible existence of extinct or dormant comets, objects that would appear asteroidal but in truth were icy objects that had their origins as comets. Kresák (1979) suggested a number of extinct comet candidates based primarily on dynamical criteria. Degewij and Tedesco (1982) compared physical studies of comets and asteroids and concluded that no extinct cometary nucleus had yet been found. Weissman et al. (1989) suggested a list of cometary candidates among the Apollo and Amor asteroids, as well as some outer solar system objects such as Chiron (later discovered to be active) and Hidalgo. Wetherill $(1988,1991)$ investigated the dynamical evolution of Jupiterfamily comets in the inner solar system and concluded that as much as half of the Apollo-Amor 
objects could be of cometary origin.

The problem is not a simple one. The chaotic nature of the dynamical evolution of objects in planet-crossing orbits, as well as nongravitational accelerations on comets caused by outgassing, make it impossible to track orbits accurately backwards (or forwards) in time more than a few decades to centuries. The obscuring effects of cometary comae make it difficult to apply the same physical observation techniques to comets that have been so successful with asteroids, when the comets are relatively close to the Earth. (Observations of cometary nuclei at large heliocentric distances when they are presumably inactive have had considerable success, e.g., Lowry 2001, but are necessarily difficult to perform and interpret.) Finally, our understanding of comets and cometary processes is still in a primitive state, and has yet to benefit from the intense scrutiny that the asteroid orbiting NEAR mission has afforded us of asteroid 433 Eros, a typical Earth-approaching, S-type asteroid. The first comet rendezvous mission, Rosetta, is scheduled to be launched in January 2003 and will arrive at periodic comet 46P/Wirtanen in November 2011.

In the past decade, several lines of research have provided new data and new tools with which to pursue these questions. The first is the extensive automated surveys for near-Earth objects (NEOs) that have serendipitously discovered many objects throughout the planetary system in unusual, sometimes comet-like orbits. Second is the advances in numerical simulation of solar system dynamics made possible by advanced numerical codes, in particular symplectic integrators, and by the availability of low-cost computers that can be dedicated to running these codes for weeks or even months. Third is the substantial increase in physical observations of comets and asteroids that permit us a better understanding of the interrelationships between these two very important classes of objects.

In this chapter we will discuss how these new tools have made it possible to argue, at least statistically, for the existence of extinct or dormant comets among the observed asteroid population, in particular among the NEOs. Our approach is considerably different from that of Weissman et al. (1989, hereafter Paper 1) in Asteroids II, and we refer the reader to that chapter for more detailed discussions of physical observations of comets and extinct/dormant comet candidates. Section 2 provides definitions of the classes of bodies we are discussing. Section 3 presents a discussion of the possible physical end-states of cometary nuclei. Section 4 discusses cometary dynamics and Section 5 reviews physical observations of comets and extinct comet candidates. Section 6 examines a number of asteroidal objects that have been identified as likely extinct comet candidates. Finally, Section 7 provides additional discussion and a summary of our results.

\section{Cometary Definitions}

Comets and asteroids represent different parts of a continuous spectrum of planetesimals that were formed and processed in the primordial solar nebula. Two parts of that "stratigraphic" record, the asteroid belt between Mars and Jupiter and the Kuiper belt beyond Neptune, have been preserved in situ, at or close to their formation locations. However, an important part of the record, the comets in and just beyond the giant planets zone, have been scattered out of that 
region by their gravitational interactions with those planets. (Many of those scattered comets are preserved in the Oort cloud.) As a result, the critical transition zone between rocky and icy objects has likely been lost from the "stratigraphic" record.

In general, the distinction between comets and asteroids has been based on the existence of a substantial fraction of ices within the object, thus providing the volatiles necessary for the object to develop a coma. Traditionally, the boundary between the formation zones for comets and asteroids has been taken to be the orbit of Jupiter at 5.2 AU, though the icy nature of several of the Galilean satellites suggests that the boundary might be placed somewhat closer to the Sun, perhaps at $\sim 4 \mathrm{AU}$.

At present, the choice between a cometary versus an asteroidal designation for a newly discovered object is based on the presence or absence of visible coma. This has occasionally led to objects being classified as both types, in particular when coma was discovered long after the object had been catalogued as an asteroid. The most notable case is the Centaur asteroid 2060 Chiron, which is also known as comet 95P/Chiron. In addition, this scheme has led to Kuiper belt objects being numbered as asteroids, though their location in the solar system argues strongly for a significant icy fraction in their compositions. Since no formal definitions of comets and asteroids exist, we will use the following, slightly modified definitions from Paper 1.

Comet (or cometary nucleus): a body formed in the outer planets region (or beyond) containing a significant fraction of volatiles in the form of ices, and capable of developing a coma if its orbit brings it close enough to the Sun.

Active comet: a comet nucleus losing volatiles and dust in a detectable coma.

Inactive comet: a comet nucleus that is active during part of its orbit, but presently is in a part of the orbit where volatile loss is negligible and there is no detectable coma.

Dormant comet: a comet nucleus which, although once active, has lost the ability to generate a detectable coma in any part of its present orbit. A dormant comet perturbed to a smaller perihelion distance might be reactivated. Or an impact might remove an overlying non-volatile crust and expose fresh icy materials to sublimation, reactivating the comet.

Extinct comet: a comet nucleus that has lost its ices, or has its ices so permanently buried under a non-volatile crust, that it is incapable of generating a coma.

Asteroid: an interplanetary body formed without significant ice content, and thus incapable of displaying cometary activity.

Near-Earth object: an asteroid or comet in an orbit with perihelion distance less than 1.3 AU. 
The informed reader will recognize exceptions and shades of grey that do not fit these definitions. For example, Kuiper belt objects, Trojan asteroids, and some outer main belt asteroids all likely have significant ice content, which would make them comets under our definition, but are officially catalogued (and often referred to) as asteroids. Also, our criteria themselves are subjective and open to interpretation. As detector technology has improved, our ability to recognize comae around distant objects has similarly improved. Also, the distinction above between dormant and extinct comets may be very subtle. If an extinct comet could somehow be split open (for example, by a catastrophic collision), exposing the ices within, would it not return to being an active comet? We do not mean the definitions to be perfect, only for them to act as a guide in our discussion below. Note that in that discussion we will use the term "extinct comet" quite frequently, but by it we mean either an extinct or dormant comet.

\section{Physical End States of Cometary Nuclei}

Comets are transient bodies, losing some fraction of their mass, perhaps $0.01-0.1 \%$, on each return. Dynamical studies of the evolution of the orbits of long-period comets have shown that the $1 / \mathrm{a}_{\mathrm{o}}$ (inverse original semi-major axis) distribution for those orbits can only be explained if there is some physical mechanism that destroys comets or renders them unobservable (Oort 1950; Whipple 1962; Weissman 1979; Bailey 1984; Wiegert and Tremaine 1999). This process is often referred to as "fading," though the actual physical mechanism or mechanisms are not identified.

In fact, compilations of observations of three Halley-type comets: 1P/Halley, 109P/SwiftTuttle, and 55P/Tempel Tuttle showed that these comets remained remarkably constant in maximum brightness over periods of $~ 600-2,200$ years (Stephenson et al, 1985; Yau et al. 1994; Yeomans et al. 1996). These periods covered 30, 17, and 19 returns for Halley, Swift-Tuttle, and Tempel-Tuttle, respectively. Similarly, Kamél (1991) showed that 2P/Encke had not faded over 155 years, encompassing 47 returns. Thus, if comets do fade, they likely do so very slowly, or episodically

This result is in contrast to that of Kresák (1987) who noted that several short-period comets had been missed on pre-discovery apparitions when the comets were close to Earth and should have achieved naked-eye brightness. Kresák proposed that these comets went through irregular dormant phases in which their activity dropped to near-zero. However, Stephenson et al. (1985), Yau et al. (1994) and Yeomans et al. (1996) showed that the three Halley-type comets noted above were not discovered by naked-eye observers unless they reached a visual magnitude of 3.5-4.0. Examination of Kresák's list of the expected brightness ranges for 14 potentially dormant comets shows that all of them could have been fainter than $\mathrm{V}=3.5-4.0$, and only 4 might have been brighter than those values. Although the total magnitude of each comet might have exceeded the threshold for naked-eye detection of stars, $\mathrm{V} \approx 6$, the diffuse nature of cometary comae in fact make them very difficult to detect unless they are significantly brighter than that threshold.

Many cases of comets that disappeared can likely be attributed to poorly determined orbits. For example, comet 107P/Wilson-Harrington was discovered in 1949 and observed for 
only 6 days, and was lost for 30 years before it was accidentally recovered in 1979 (see Section $6)$. But in other cases, well-observed comets seem to have simply disappeared. These include comet 3D/Biela (observed 1772-1852, see below), 5D/Brorsen (1846-1879), 11D/Tempel-Swift (1869-1908), 18D/Perrine-Mrkos (1896-1968), 20D/Westphal (1852-1913), 25D/Neujmin 2 (1916-1926), and 34D/Gale (1927-1938). Note that the designation "D" refers to "defunct" comets. Several of these comets displayed unusual brightness changes on their last apparition (outbursts and/or fading), perhaps indicative of their imminent demise (Kronk, 1984).

Three physical mechanisms have been proposed to explain the disappearance of comets: 1) random disruption, 2) loss of all volatiles, and 3) formation of a non-volatile crust or mantle on the nucleus surface. Unfortunately, none of these mechanisms are well understood or quantified. Nevertheless, some physical loss mechanism(s) is needed to match the energy distribution of the orbits of the known long-period comets. Weissman (1979) achieved this by assuming that comets split destructively at a rate of $\sim 12 \%$ per perihelion passage, but that only $85 \%$ of comets were capable of splitting. Wiegert and Tremaine (1999) proposed that: 1) the fraction of comets remaining visible after $\mathrm{m}$ apparitions is proportional to $\mathrm{m}^{-0.6 \pm 0.1}$, or 2 ) $\sim 95 \%$ of comets live for only $\sim 6$ returns and the remainder last indefinitely.

Random disruption of comets has been observed on many occasions. The classic example is comet 3D/Biela, a Jupiter-family comet with a period of 6.6 years that was observed in $1772,1805,1826$, and 1832. The comet was observed to split during its 1846 apparition, and returned as a double comet in 1852. It was never observed again. More recently Comet LINEAR, D/1999 S4, was observed to completely disrupt as it passed through perihelion in July 2000 (Weaver et al. 2000; see Figure 1).

Weissman (1980) compiled records of observations of disrupted or split comets and showed that $10 \%$ of dynamically new comets from the Oort cloud split, versus $4 \%$ for returning long-period comets, and only 1\% for short-period comets (per orbit; see also Sekanina 1982). The splitting events did not show any correlation with perihelion distance, distance from the ecliptic plane, or time of perihelion passage. The statistics suggest that the tendency of cometary nuclei to split may reflect some intrinsic property, such that comets that are likely to split do so early on, and those that are unlikely to split are able to survive for hundreds or even thousands of returns.

Note that splitting events do not always lead to total disruption of the nucleus. For example, Comet 73P/Schwassmann-Wachmann 3 has been observed to shed fragments on at least three perihelion passages, yet still returns every 5.3 years. Also, since splitting appears to eventually lead to destruction of the cometary nucleus, it cannot explain dormant or extinct nuclei that might be observed as asteroidal objects.

Loss of all volatiles is a slowly acting process. Weissman (1980) estimated lifetimes of $\sim 600,4,500$, and $4 \times 10^{5}$ returns for $1-\mathrm{km}$ radius water ice spheres with surface albedo $=0$ and density of $0.6 \mathrm{~g} \mathrm{~cm}^{-3}$, for long-period comets with perihelia of 1, 2, and $3 \mathrm{AU}$, respectively. (Lifetimes for icy spheres in short-period comet orbits with comparable perihelia would be similar though somewhat shorter because of their less eccentric orbits.) However, it is not clear that a comet could sublimate all of its volatiles, leaving a coherent, non-volatile remnant nucleus. 
Evolving gases would tend to carry away much of the non-volatile matter, and any residual material would form an insulating layer that would prevent insolation from penetrating to the volatiles at depth. Thus, total loss of volatiles appears to be an unlikely end-state, or one that would not leave a substantial extinct nucleus.

Formation of a non-volatile crust or mantle on the nucleus surface, first proposed by Whipple (1950), is the one process that would presumably lead to asteroid-like objects. There are two ways that such crusts may form. First, irradiation of comets by galactic cosmic rays and solar protons will sputter away volatiles and transform organic molecules to more refractory forms during the comets' long storage in the Oort cloud or the Kuiper belt (Johnson et al. 1987; Moore et al. 1983; see also Weissman and Stern 1997 and references therein). This irradiated crust would extend $\sim 1 / \rho$ meters below the nucleus surface, where $\rho$ is the density of the cometary materials in $\mathrm{g} \mathrm{cm}^{-3}$. An interesting and unsolved problem is how such a crust is removed when the comet re-enters the planetary region on it first perihelion passage, allowing it to become active.

A second method for forming a cometary surface crust is a lag deposit of non-volatile grains, left behind or launched on suborbital trajectories that do not achieve escape velocity, as water and more volatile ices sublimate from the nucleus surface during perihelion passage. It is not clear how such large non-volatile grains, which presumably are too heavy to be lifted off the nucleus by evolving gases, might form. However, if they did, thermal models have shown that a layer only a few to perhaps $10 \mathrm{~cm}$ thick would be sufficient to insulate the underlying ices from sublimation (Brin and Mendis 1979; Fanale and Salvail 1984; Prialnik and Bar-Nun 1988).

The existence of crusts, or more specifically, inactive areas on cometary nuclei, was dramatically shown by the spacecraft images of Comet Halley in 1986 (Figure 2, Keller et al. 1986) and again by images of the nucleus of Comet Borrelly in 2001. Dust jets emanated from distinct and relatively small regions on the nucleus surface. From the Halley images, it was estimated that only $10 \%$ of the visible surface, or $\sim 20-30 \%$ of the sunlit surface was active. This agreed well with thermal modeling of the water production from Halley which showed that only $30 \%$ of the sunlit surface needed to be active to match the total water production rate (Weissman 1987). At the time of this writing, the Borrelly images are still undergoing analysis, but the apparently active regions are again, fairly small. Although one cannot say for certain that the apparently inactive areas in Figure 2 are not emitting gas (dust emission can likely be ruled out as it would be visible), the Halley and Borrelly images appear to support the idea that comets can develop crusts and thus evolve to dormant or extinct objects.

Further support comes from estimates of the active fraction of other cometary nuclei. A'Hearn (1988) showed that many Jupiter-family comets have active fractions of only a few percent. For comets 49P/Arend-Rigaux and 28P/Neujmin 1, both of which are trapped in dynamical resonances that have prolonged their lifetimes in the active comets region (Marsden 1971), the active fractions are estimated as only $0.08 \%$ and $0.1 \%$, respectively! This suggests that comets may slowly age and evolve towards total inactivity, either by developing nonvolatile crusts on their surfaces or by some other as yet unrecognized mechanism. Thus, crust formation does appear to provide a mechanism for evolving cometary nuclei to dormant or extinct states. 


\section{Cometary Dynamics: Evolution to Asteroidal Orbits}

Comets have traditionally been divided into two major dynamical groups: long-period (LP) comets with orbital periods > 200 years, and short-period (SP) comets with periods $<200$ years. In addition, long-period comets typically have random orbital inclinations while shortperiod comets typically have inclinations relatively close, within $\sim 35^{\circ}$, to the ecliptic plane. In the past decade it has become common to break the SP comets into two sub-groups: Jupiterfamily comets (JFC) with periods less than 20 years and a median inclination of $\sim 11^{\circ}$, and Halley-type comets (HTC) with periods between 20 and 200 years and a median inclination of $\sim 45^{\circ}$.

A more formal dynamical definition of the difference between the JFC and HTC comets was proposed by Carusi and Valsecchi (1987) based on the Tisserand parameter. The Tisserand parameter is an approximation to the Jacobi constant, which is an integral of the motion in the circular restricted three-body problem. It was originally devised to recognize returning periodic comets that may have been perturbed by Jupiter and is given by

$$
\mathrm{T}=\mathrm{a}_{\mathrm{J}} / \mathrm{a}+2 \sqrt{\left(\mathrm{a} / \mathrm{a}_{\mathrm{J}}\right)\left(1-\mathrm{e}^{2}\right)} \cos (\mathrm{i})
$$

where $a_{J}$ is Jupiter's semi-major axis, and a, e, and $i$ are the comet's semi-major axis, eccentricity, and inclination, respectively. T is also a measure of the relative velocity between a comet and Jupiter during close encounters:

$$
\mathrm{v}_{\mathrm{rel}}=\mathrm{v}_{\mathrm{J}} \sqrt{3-\mathrm{T}}
$$

where $\mathrm{v}_{\mathrm{J}}$ is Jupiter's circular orbit velocity about the Sun. Objects with T close to, but smaller than 3 have very slow, and thus very strong, encounters with Jupiter. Objects with $T>3$ are not Jupiter-crossing.

Jupiter-family comets have $\mathrm{T}>2$ while long-period and Halley-type comets have $\mathrm{T}<2$. Levison (1996) suggested a new nomenclature that recognized this new classification scheme. He proposed that comets with $\mathrm{T}>2$ be known as ecliptic comets while comets with $\mathrm{T}<2$ be known as nearly-isotropic comets. We will use this nomenclature in the discussions that follow.

The different dynamical regimes occupied by comets and asteroids are seen in Figure 3, which is a scatter diagram in semi-major axis and eccentricity of the known asteroids brighter than $\mathrm{H}=18$ (corresponding to $\sim 1 \mathrm{~km}$ diameter objects) and all known ecliptic comets. The various lines in the diagram depict lines of constant perihelion or aphelion distance for $\mathrm{q}=1.017$ and 1.3 AU, and $\mathrm{Q}=0.983 \mathrm{AU}$, and lines of constant $\mathrm{T}=2$ and 3 . Objects in the center of the main belt have been removed for clarity. The orbital data is taken from the Minor Planet Center file of orbits for March 8, 2001 (cfa-ftp.harvard.edu/pub/MPCORB/MPCORB.DAT and COMET.DAT). Asteroids are plotted as filled circles in Figure 3, comets as open circles.

The effect of the $\mathrm{T}=3$ barrier is clearly seen. It is difficult for ecliptic comets to dynamically detach themselves from Jupiter and evolve to orbits with $\mathrm{T}>3$. The only real 
exception is Comet 2P/Encke ( $\left.\mathrm{a}=2.217 \mathrm{AU}, \mathrm{e}=0.847, \mathrm{i}=11.8^{\circ}, \mathrm{T}=3.026\right)$, which will be discussed below. In total, $11 \mathrm{JFC}$ comets have orbits with $\mathrm{T}>3$; most are sufficiently Jupiterapproaching (with aphelia very close to or crossing Jupiter's eccentric orbit) that they are still under the gravitational influence of Jupiter. Also, Figure 3 is slightly misleading as it attempts to plot a three-dimensional function, $\mathrm{T}(\mathrm{a}, \mathrm{e}, \mathrm{i})$ on a two-dimensional, $(\mathrm{a}, \mathrm{e})$ plane.

Asteroids, for the most part, have $\mathrm{T}>3$; the Jupiter Trojans are of course a notable exception. Historically, the failure to find asteroidal-appearing bodies with $\mathrm{T}<3$ was used as an argument against the existence of extinct cometary nuclei. However, in the mid 1980's search programs began to find these asteroids; many of these can be seen in Figure 3. As we will see below, it is now possible to argue on statistical grounds which of these objects have a high probability of being derived from ecliptic comets.

Comets on planet-crossing orbits are transient members of the solar system. Close and/or distant encounters with the giant planets, in particular Jupiter, limit their mean dynamical lifetimes to 0.4-0.6 Myr (Weissman 1979; Levison and Duncan 1997). Thus, they must be continually resupplied from long-lived dynamical reservoirs. The different inclination distributions of the ecliptic and nearly-isotropic comets reflect their different source reservoirs. Nearly-isotropic comets (LP and HTC) are believed to originate from the nearly-spherical Oort cloud, the vast cloud of some $10^{12}$ to $10^{13}$ comets surrounding the solar system at distances of $10^{3}$ to $10^{5} \mathrm{AU}$ (Oort 1950; for a review see Weissman 1996a). Ecliptic comets are fed into the planetary system from the highly flattened Kuiper belt of some $10^{9}$ to $10^{10}$ comets beyond the orbit of Neptune, extending from $~ 35$ to perhaps several hundred AU (for reviews see Weissman and Levison 1997, Malhotra et al. 2000). A third reservoir that may supply ecliptic comets to the planetary region is the scattered disk (Duncan and Levison 1997), icy planetesimals scattered out of the Uranus-Neptune zone and inner Kuiper belt to large semi-major axes, several hundred to $\sim 1,000 \mathrm{AU}$ (though with perihelia still close to Neptune's orbit), but not large enough for them to be captured into the Oort cloud.

Much of the research into cometary dynamics in recent years has had the goal of understanding the structure and evolution of these mostly invisible reservoirs. These reservoirs were formed at the same time as the planets. Indeed, they can be viewed as the remnants of planet formation. We discuss the evolution of comets from each of these reservoirs to asteroidal orbits below.

\section{Ecliptic Comets}

The observed ecliptic comets have a very flattened inclination distribution; the Jupiterfamily comets have a median inclination of only $11^{\circ}$. In the last 15 years, research attempting to explain this inclination distribution has been extremely active. Indeed, attempting to understand these comets led to one of the most important discoveries in planetary science in the second half of the 20th century - the discovery of the Kuiper belt.

Historically, the ecliptic comets were thought to originate from nearly-isotropic comets that had been captured into short-period orbits by gravitational encounters with the planets (Newton 1891, 1893; Everhart 1977). Fernández (1980) argued that this process is too 
inefficient and suggested that a belt of distant icy planetesimals beyond Neptune could better serve as the source of most of the ecliptic comets; such a belt was suggested by Kuiper (1951) and later investigated by Whipple (1964). Duncan et al. (1988) greatly strengthened this argument by performing dynamical simulations which showed that a cometary source beyond Neptune with a low initial inclination distribution (which they named the Kuiper belt) was far more consistent with the observed orbits of the JFC comets than the randomly distributed inclinations of comets in the Oort cloud (see also Quinn et al. 1990). The first Kuiper belt object, 1992 QB $_{1}$ (now numbered asteroid 15760), was discovered in 1992 (Luu and Jewitt 1993). Since then over 460 objects have been discovered in orbits beyond Neptune.

A second potential source of ecliptic comets is the scattered disk, predicted by Duncan and Levison (1997). Although the scattered disk is related to the Kuiper belt, it is dynamically distinct, consisting of objects in highly eccentric and inclined orbits that are typically Neptunecrossing or approaching. Approximately 50 scattered disk objects have now been found. However, once an object leaves the Kuiper belt or the scattered disk, its dynamical evolution is indistinguishable. Thus, for the remainder of this discussion, we will not distinguish between these two source regions.

Levison and Duncan (1997, hereafter LD97) have presented the most comprehensive simulations to date of the dynamical evolution of objects from the Kuiper belt. They found that as objects evolve inward from the Kuiper belt or scattered disk, they tend to be under the dynamical control of only one planet at any time, and the Tisserand parameter with respect to that planet is always close to 3 . The planet scatters the comets randomly inward and outward. However, the comets can reach maximum eccentricities on the order of only $\sim 0.25$ due to the large Tisserand parameter. Thus, the planet can only hand the comets off to the planet directly interior or exterior to it. However, once the comets come under the gravitation influence of Jupiter, they can have much larger eccentricities, even up to 1.0, at which point they can be ejected from the solar system.

LD97 estimated the ratio of active to extinct ecliptic comets by comparing the orbital inclination distribution of simulated JFCs to that of the observed JFCs. LD97 estimated the physical lifetimes of JFCs, restricting the analysis to JFCs with perihelion distances $<2.5 \mathrm{AU}$ and absolute cometary magnitudes, $\mathrm{H}_{10}$, brighter than 9 , which they argued are observationally complete. (The cometary absolute magnitude, $\mathrm{H}_{10}$, is the total magnitude the active comet would have at $1 \mathrm{AU}$ from the Sun and $1 \mathrm{AU}$ from the Earth, assuming that cometary brightness varies with heliocentric distance, $\mathrm{r}$, as $\mathrm{r}^{-4}$. Note that this is different from the traditional $\mathrm{H}$ magnitude system for asteroids.)

As described above, the observed JFCs have a very flattened inclination distribution with a median inclination of only $11^{\circ}$. However, LD97 showed that the inclinations were even lower when objects were first injected into this population and subsequently increased as a function of time (see also Levison and Duncan 1994). This can be seen in Figure 4. The heavy solid curve in Figure 4 shows the cumulative inclination distribution of the known JFCs, while the top dotted curve shows the cumulative inclination distribution of LD97's simulated comets when they were first injected into the visible JFC region, q $<2.5 \mathrm{AU}$. As can be seen in the Figure, the median inclination of the model is significantly smaller than the observations. A Kolmogorov-Smirnov 
test shows that the probability that the two distributions are derived from the same parent distribution is only $7 \times 10^{-3}$.

The bottom dotted curve in Figure 4 shows the inclination distribution that LD97 predicts if the JFCs are in steady-state dynamical equilibrium and if they remain active throughout their time in the JFC region. In this case the median inclination is significantly larger than is observed. This implies that we are not seeing all of the comets in the JFC population, and that the dynamically older comets are remaining undetected or unrecognized as comets. Or, some physical mechanism is removing the comets before they can reach dynamical maturity.

Indeed, it is possible to match the model to the observations by assuming a simple model for the physical aging of comets. LD97 modeled the effects of physical aging by assuming that all comets remain active for $t_{\mathrm{e}}$ years, after which they are permanently extinct. They found good agreement to the observed inclination distribution of JFCs for values of $t_{\mathrm{e}}$ between 3,000 and 25,000 years. The most likely value is 12,000 years, or about 1,600 perihelion passages, assuming a typical orbital period of $\sim 7.5$ years. The dashed curve in Figure 4 shows the predicted inclination distribution for $t_{e}=12,000$ years. The match to the observations is excellent. A Kolmogorov-Smirnov test shows that the probability that the two distributions are derived from the same parent distribution is $>0.9$.

The fact that LD97 needed to invoke a physical lifetime for comets that is significantly smaller than their dynamical lifetime $\left(2.7 \times 10^{5}\right.$ years, though much of that time is spent at perihelia $>2.5 \mathrm{AU}$ ) suggests that there may be a significant number of extinct comets in orbits similar to the JFCs. LD97 estimated the ratio of extinct to active JFCs with perihelion distance < 2.5 AU. For values of $t_{\mathrm{e}}$ between 3,000 and 25,000 years, this ratio is between 7 and 2. If one assumes $t_{\mathrm{e}}=12,000$ years, then the ratio is 3.5. Based on data from Fernández et al. (1992), LD97 estimated that there are 108 active JFCs with $\mathrm{q}<2.5 \mathrm{AU}$ and $\mathrm{H}_{10}<9$ in the solar system. Thus, there may be $\sim 380$ extinct JFCs with $\mathrm{q}<2.5 \mathrm{AU}$ and $\mathrm{H}_{10}<9$.

There are several caveats that must be understood before interpreting this result. First, the above estimates assume that all comets become extinct rather than disintegrating. As discussed in the previous section, splitting or disruption may be a common end-state for ecliptic comets. Thus, we must assume that the above estimate is an upper limit to the population of extinct JFCs.

Also, LD97's integrations failed to produce objects with orbits similar to that of Comet 2P/Encke. P/Encke is a bright, low-inclination comet whose orbit is distinguished by an aphelion distance of only 4.1 AU, well inside the orbit of Jupiter. Although similar to JFCs in all other respects, 2P/Encke is dynamically decoupled from Jupiter. LD97 most likely did not produce objects on orbits similar to this comet because their integrations did not include the terrestrial planets or the effects of nongravitational forces (forces from outgassing on the dayside of the rotating cometary nucleus). So, the above estimate of the extinct comets does not include this population.

Harris and Bailey (1998) performed dynamical integrations that did include the terrestrial planets and nongravitational forces, and showed that the capture rate into decoupled, Encke-like 
orbits was substantially increased. They found that nongravitational forces, in particular, substantially enhanced the capture rate into decoupled orbits, if the nongrav forces acted in a uniform manner throughout the comet's dynamical evolution. However, the latter may not be the case as there is evidence for apparent precession of cometary rotation pole orientations and for variations in the nongravitational accelerations (see, e.g., Sekanina 1991). Also, Weissman (1979) showed that uniformly acting nongrav forces led to predictions for the long-period comet population that were not, in fact, observed.

Finally, the estimate of $\sim 380$ extinct JFCs refers to objects that at one time had absolute magnitudes brighter than $\mathrm{H}_{10}=9$. Ideally, we would like this estimate to be with respect to objects larger than some known size. Relationships that equate $\mathrm{H}_{10}$ with nucleus size have been proposed by Weissman (1990) and Bailey and Stagg (1988) but others have found such relationships poorly founded and unreliable (Zahnle et al. 1998, Levison et al. 2000).

A new method for estimating the number of extinct JFCs is now possible based on studies of the origin of the NEO population. Bottke et al. (2002) modeled the orbital and absolute magnitude distributions of the NEO population, using 138 discoveries or accidental rediscoveries from a single source, the Spacewatch survey (Scotti 1996), and then correcting for observational selection effects. The details of this modeling effort are reviewed in the chapter by Morbidelli et al.; we will summarize them here.

Bottke et al. numerically integrated thousands of test particles from five source regions that they believed provided most NEOs to the terrestrial planets region. These source regions are: 1) the $v_{6}$ secular resonance at $\sim 2.1 \mathrm{AU} ; 2$ ) the Mars-crossing asteroid population adjacent to the main belt; 3) the 3:1 mean-motion resonance with Jupiter at $2.5 \mathrm{AU} ; 4$ ) the outer main belt population between 2.8 and 3.5 AU; and 5) the Jupiter-family comets. The dynamical simulation of the JFCs used the integrations of LD97.

By comparing the orbital element distributions of hypothetical NEOs originating from these five regions to the observed NEO orbital distributions derived from the Spacewatch survey, Bottke et al. estimated the relative importance of each NEO source region. Although there is considerable overlap between the NEOs produced by the individual source regions, particularly for NEOs with semimajor axes < $2.8 \mathrm{AU}$, the JFC's produced NEO's that were largely distinct from the other source regions, with the exception of the outer main belt asteroid source region. JFC-derived NEOs had semi-major axes $>2.6 \mathrm{AU}$ and eccentricities $>0.55$.

Bottke et al. found that $\sim 6 \% \pm 4 \%$ of NEOs with a $<7.4 \mathrm{AU}, \mathrm{e}<1.0, \mathrm{i}<90^{\circ}$, and $13<\mathrm{H}$ $<22$ are extinct JFCs. ( $\mathrm{H}$ in this case refers to the standard absolute magnitude system for asteroids). The total number of NEOs with $\mathrm{H}<18$ was found to be $\sim 960 \pm 120$. These results imply that there are $\sim 61 \pm 50$ extinct JFCs with $\mathrm{H}<18$ among the NEO population.

If we assume that an extinct comet has an albedo of 0.04 , like those measured for cometary nuclei (Fernández 1999), we obtain a diameter of $1.7 \mathrm{~km}$ for $\mathrm{H}=18$ (Bowell et al. 1989). Shoemaker and Wolfe (1982) estimated that the cumulative cometary nucleus size distribution has the form $\mathrm{N}(>\mathrm{D}) \propto \mathrm{D}^{-2.2}$, though Lowry (2001) more recently found a shallower slope of -1.6; Weissman and Lowry (2001) extended the latter work and found a slope of -1.4. 
In contrast, Fernández et al. (1999) found a slope of -2.65 , though the sample they fit was quite small, only 9 comets. Thus, the number of 1-km diameter extinct JFCs among the NEOs $(\mathrm{q}<1.3$ AU) is likely $\sim 140$ to 200 objects. Bottke et al. (2002), using their estimated NEO size distribution of $\mathrm{N}(>\mathrm{D}) \propto \mathrm{D}^{-1.75}$, calculated that there were $\sim 1501-\mathrm{km}$ diameter extinct JFCs among the NEOs. If one assumes a uniform perihelion distribution versus heliocentric distance, these numbers compare favorably with the LD97 estimate of $~ 380$ extinct JFCs with q $<2.5$ AU (though we do not know if we are talking about the same size objects).

Bottke et al. also used their model to estimate the probability that any individual known NEO is an extinct comet, based on its semi-major axis, eccentricity, and inclination. Taking the list of asteroids from the November 2000 database of T. Bowell (http://asteroid.lowell.edu), Bottke et al. found 57 NEOs with a $\mathrm{P}_{\mathrm{JFC}}>10 \%$ chance of having originally been an ecliptic comet. These objects are listed in Table 1 , in order of decreasing $\mathrm{P}_{\mathrm{JFC}}$. In addition to the orbital elements a, e, and i for each object, Table 1 lists the Tisserand parameter for that orbit, and the probability that the object comes from each of the five source regions used by Bottke et al. (The last column, which refers to the object's spectral type and/or albedo, will be discussed in the next Section.) Note that as one reads down the Table, the most probable source region shifts from the JFC population to the outer main belt, and then to asteroid regions at successively smaller semimajor axes. Most of the objects in Table 1 have $2<\mathrm{T}<3$. A plot of the (a,e) positions of the 57 objects listed in Table 1 is shown in Figure 5.

There are several caveats that must be noted with regard to the work of Bottke et al. (2002). First, the results quoted are for Bottke et al.'s best-fit model, and the solution may not be unique. Second, Bottke et al. did not include the nearly-isotropic comets as a potential source of NEOs. Third, the integrations of the orbits of the JFCs did not include the terrestrial planets (which were included for all of the asteroid source region integrations) or nongravitational forces. The inclusion of the terrestrial planets and nongrav forces would likely have resulted in more comets being captured to smaller semi-major axes, in particular to orbits dynamically detached from Jupiter, and this would likely have increased the fraction of NEOs that may have originated from the JFC population.

\section{Nearly-Isotropic Comets}

Nearly-isotropic comets (NICs) are believed to originate from the Oort cloud. Monte Carlo simulations by Weissman (1979) showed that the typical long-period comet from the Oort cloud makes an average of 5 perihelion passages through the planetary region (for perihelia less than 4 AU) with a mean time of $0.6 \mathrm{Myr}$ between the first and last passage. Approximately $65 \%$ of LP comets are dynamically ejected to interstellar space (primarily by Jupiter), 27\% are lost to random disruption (or some other physical mechanism), and the remainder are lost to a variety of processes including loss of all volatiles or perturbation onto a Sun-impacting orbit. The mean semi-major axis of an observed "dynamically new" LP comet (a comet passing through the planetary region for the first time) is $22,000 \mathrm{AU}$ (Marsden et al. 1978).

Oort cloud comets have been stored at great distances from the Sun for most of the history of the solar system. However, it is most likely that the Oort cloud comets were formed in the giant planets region (Kuiper 1951). That is, the comets were formed as icy planetesimals in 
nearly circular orbits between and beyond the giant planets and are the probable remnants of planetary formation (Duncan et al. 1987; Fernández 1997). As the giant planets grew, the comets were gravitationally scattered to larger semi-major axes. When the semi-major axes had grown sufficiently, to several thousand AU, perturbations by the tidal field of the galactic disk and by random passing stars scattered the perihelia of the cometary orbits, raising many of them away from the planetary region. Comets from the Oort cloud are currently being fed back into the planetary region by these same galactic and stellar perturbations.

Dynamical modeling of the Oort cloud (Duncan et al. 1987; Dones et al. 2000) suggests that there are two dynamical regimes within the cloud. The outer, dynamically active cloud, as first described by Oort (1950), is approximately spherical and comets there are fed directly into the planetary system by the perturbations of the galactic tide and random passing stars. These are the long-period comets that we see. The outer Oort cloud reaches from $\sim 15,000$ to 100,000 AU (in semi-major axis), though as noted above, most observed LP comets have semi-major axes of $\sim 20,000-30,000$ AU. Cometary orbits in the outer cloud have been randomized by stellar and galactic perturbations, with random inclinations and a mean eccentricity of $\sim 0.7$ (Hills 1981).

The inner Oort cloud stretches from $~ 3,000$ to 15,000 AU in semi-major axis (Duncan et al. 1987). The inner cloud is dynamically less active because, being closer to the Sun, it does not feel the tidal effect of the galactic disk and random passing stars. An exception to this occurs if a passing star penetrates the Oort cloud (Hills 1981, Weissman 1996b), in which case large numbers of comets may be perturbed into the planetary region, resulting in a "cometary shower." The best current evidence suggests that we are not presently in a cometary shower (Fernández and Ip 1991; Weissman 1993). Because it is not subject to the same external perturbations as the outer cloud, cometary orbits in the inner Oort cloud have not been completely randomized and the inner cloud is believed to be flattened, with inclinations relatively close, within $\sim 45^{\circ}$ of the ecliptic plane.

Weissman (1996a) estimated a population of $10^{12}$ comets in the outer Oort cloud, brighter than $\mathrm{H}_{10}=11$, by comparing dynamical models with the observed flux of LP comets through the planetary system. Dones et al. (2000) found that the populations of the inner and outer clouds were roughly equal. However, that model assumed that the Oort cloud formed in a galactic environment similar to the one that the solar system is in today. The inner Oort cloud could contain a significantly larger population if the Sun formed in a star cluster or molecular cloud, which seems likely (Fernández 1997; Eggers 1999; Fernández and Brunini 2000). Also, Levison et al. (2001) argued that a massive inner Oort cloud must exist in order to explain the observed Halley-type comet population. Thus, the population of the inner Oort cloud is very uncertain but is thought to be at least a few times $10^{12}$ comets.

Galactic tides and stellar perturbations change the perihelion distances of Oort cloud comets, feeding them into (and out of) the planetary region. As a comet comes through the planetary system for the first time, it receives a random kick in orbital energy that is, on average (if it is Saturn or Jupiter-crossing), much larger than its binding energy. Thus, depending on the sign of the kick, the comet is either ejected from the solar system or captured onto an orbit with a smaller semi-major axis (and removed from the realm where galactic and stellar perturbations 
are important). This random walk in orbital energy continues until the comet reaches some dynamical or physical end-state.

There are two dynamical paths that an Oort cloud comet can take in order to become a visible comet. Comets from the outer Oort cloud can be perturbed from orbits with perihelia beyond the orbit of Neptune to perihelia inside the orbit of Jupiter. This involves a substantial decrease in the angular momentum of the orbit. Hills (1981; also see Duncan et al. 1987) showed that the rate of change in a comet's perihelion distance due to galactic tides is proportional to $\mathrm{a}^{2}$, where a is the orbit semi-major axis. Thus, only orbits sufficiently distant from the Sun feel the strong tidal effects of galactic and stellar perturbations, and can achieve this direct perturbation into the visible region. This is why the outer Oort cloud is dynamically active and the inner cloud is less so.

If a comet is not perturbed to a perihelion distance inside Jupiter's orbit, it will still undergo a random walk in energy due to perturbations by the giant planets, but likely without ever becoming visible. We note that some NICs have been discovered with perihelia beyond Jupiter; these are always dynamically new comets, which are known to be anomalously bright on their first perihelion passage (see Weissman 1996a for a discussion of possible reasons). This inability for comets to evolve into the inner planets region unless they are thrown there directly has been called the "Jupiter barrier" and has been examined by Weissman (1985), Wetherill (1994) and others.

However, comets from both the inner and outer clouds can follow a second path into the visible region. As noted above, comets that are thrown into orbits with perihelia among the giant planets undergo an orbital evolution that can lead to some of them being captured to intermediate-period, Halley-type orbits. Because this evolution occurs relatively far from the Sun, beyond the water ice sublimation region, the comets may not be subjected to the same insolation-related physical loss mechanisms as those with perihelia inside the orbit of Jupiter. This dynamical path was first proposed by Everhart (1977) as a possible source of Jupiter-family comets.

As with the ecliptic comets, a diagnostic for the origin of the HTCs is their inclination distribution (Levison et al. 2001, hereafter LDD01). The cumulative inclination distributions of LP comets (dotted line), HTCs (dashed line), and JFCs (solid line), taken from Marsden and Williams' (1999) catalog, is shown in Figure 6. While the distribution of the LP comets is isotropic, the HTCs are mainly prograde with a median inclination of only $45^{\circ}$, and the JFCs are all prograde with a median inclination of only $11^{\circ}$.

The most complete work on the subject of the HTC inclination distribution was performed by LDD01. They integrated the orbits of 27,700 test particles initially entering the planetary system from the Oort cloud. They found that an isotropically distributed Oort cloud does not reproduce the observed orbital element distribution of the HTCs. In order to match the observations, the initial inclination distribution of the progenitors of the HTCs must be similar to the observed HTC inclination distribution. LDD01 could match the observations with an Oort cloud that consists of an isotropic outer cloud and a massive disk-like inner cloud. Their best fit model consists of an inner Oort cloud with a median inclination of only $20^{\circ}$. 
However, LDD01's model predicts too many observed HTCs as compared to the observed number of dynamically new long-period comets. This inconsistency may be related to the fading described in Section 3 for the LP comets, though LDD01's model requires a more sever fading law than has been suggested previously. Thus, the origin of the Halley-type comets remains problematic.

If physical loss mechanisms do produce extinct LP and HTC nuclei, rather than simply destroying them, then it may be possible to find such objects among the asteroid population. These objects should have Tisserand parameters, $\mathrm{T}<2$, and semi-major axes outside of the radius of Jupiter's orbit. They also are likely to be in high inclination orbits.

It is also important to be able to show that these objects cannot have an asteroidal origin. Bottke et al.'s (2002) integrations show that when objects leaving the asteroid belt cross the orbit of Jupiter, they have $\mathrm{T}>2$. Further, LD97's integrations show that objects with $\mathrm{T}>2$ are unlikely to evolve onto orbits similar to visible HTCs or LP comets.

Table 2 lists 11 asteroids from the Minor Planet Center database (July 27, 2001) with semi-major-axes $>8 \mathrm{AU}$ and eccentricities $>0.4$. Dynamically, it is most probable that these objects are all derived from NIC-type orbits, and not from the asteroid belt. Note the large number of high inclination objects among the sample. The median inclination of these objects is $62^{\circ}$, which is close enough to that of the observed HTCs, given the small number of objects in both samples, to suggest that they may be the same.

Given that we believe that the objects in Table 2 came from the Oort cloud, can we conclude that they are extinct comets? Unfortunately not. Weissman and Levison (1997) examined the orbit of 1996 PW and showed that it was almost certainly derived from the Oort cloud. But they found that the probability of it being an extinct cometary nucleus was roughly equal to the probability of it being an asteroid ejected during the clearing of the planetary zones in the early solar system. Hicks et al. (2000) found D-type colors for 1996 PW, consistent with it being either an extinct nucleus or an outer main belt asteroid. Weissman and Levison proposed that $\sim 0.8-2.3 \%$ of the Oort cloud population is likely asteroidal, material ejected from the outer primordial asteroid belt and from dynamical resonances during the late stages of planetary zone clearing. These objects would most likely be indistinguishable from extinct comets, using Earth-based observations, except perhaps by their spectral types (see Section 5). Thus, we are unable to determine with certainty if an asteroidal-appearing object in a NIC-like orbit is an extinct comet or an asteroid from the Oort cloud.

\section{Physical Studies of Cometary Nuclei: Comparison with Asteroids}

The same techniques that can be used to study and classify asteroids, e.g., BVRI photometry, reflection spectroscopy, thermal IR observations, etc, have now begun to be applied to cometary nuclei. Observations of "bare" cometary nuclei are necessarily difficult because they must be performed when the comets are far from the Sun and apparently inactive, and thus relatively faint when observed from Earth. For example, a bare nucleus with a radius of $2 \mathrm{~km}$ and an albedo of 0.04 would be V magnitude $20.0,21.5$ and 22.6 at 3, 4, and 5 AU, 
respectively, at opposition. Also, it is difficult to rule out the presence of unresolved coma in such studies.

Nevertheless, a consistent picture of cometary nuclei has begun to emerge. Nuclei are typically elongated bodies, a few kilometers in radius, with surface albedos of $0.04 \pm 0.02$ (see Meech, in press, and references therein, also Fernández, 1999). Their visible spectra are typically featureless and range from grey to very red objects, similar to C or D-type asteroids (Luu 1993; see also Paper 1, Figure 2). The distribution of rotation periods tends to be similar but somewhat slower than main-belt asteroids; there are no recognized fast rotators among the cometary nuclei. The latter is suggestive of low tensile strengths for the cometary nuclei. Bulk densities for cometary nuclei have only been estimated indirectly, based either on the effect of nongravitational forces on the orbit of comet 1P/Halley: $0.2-1.3 \mathrm{~g} \mathrm{~cm}^{-3}$ (Rickman 1986, Peale 1989), or on the tidal disruption and reassembly of the fragments of comet D/Shoemaker-Levy 9: $0.6-1.1 \mathrm{~g} \mathrm{~cm}^{-3}$ (Asphaug \& Benz 1994, 1996). Preferred models of nuclei suggest that they are "primordial rubble piles" or "fluffy aggregates," (Weissman 1986; Donn and Hughes 1986), having received relatively little physical processing since they accreted in the solar nebula 4.5 Gyr ago. The spacecraft images of Comets Halley and Borrelly, shown in Figure 2, are consistent with this description. The topography of the nucleus surfaces appears to be chaotic and rough at all scales.

Although none of these properties provide absolute discriminators between comets and asteroids, we can test Bottke et al.'s (2002) sample of extinct cometary candidates, listed in Table 1, for consistency with a cometary origin. The final column of Table 1 lists the identified spectral types (and/or measured albedos) of 20 of the candidate comets, drawn from the literature and from personal communications with M. Hicks, R. Whiteley, B. Bus, R. Binzel, and Y. Fernández. We find that those objects with $\mathrm{P}_{\mathrm{JFC}}>0.5$ are dominated by primitive spectral types; 6 out of 7 observed objects are spectral type C, D, F, or low albedo (see below). On the other hand, only 5 out of 13 observed objects with $\mathrm{P}_{\mathrm{JFC}}<0.5$ are primitive types. Thus, Bottke et al.'s dynamical argument for many of these objects being extinct comets is independently substantiated by the spectral data. We emphasize that although this is not a proof that these objects are cometary, it is consistent with them having a cometary origin.

Similarly, Fernández et al. (2001) compared measured albedos for 14 cometary nuclei and 10 NEOs with Tisserand parameters $<3$, and 34 NEOs with $\mathrm{T}>3$. They showed that all of the comets and 9 out of 10 of the NEOs with $\mathrm{T}<3$ had low albedos, $\leq 0.07$, while most of the NEOs with $\mathrm{T}>3$ had albedos $>0.15$. Again, this is consistent with the $\mathrm{T}<3$ objects having a cometary origin.

In Table 2 we list the spectral types or measured albedos for 5 of the 11 Oort cloud candidates. All of these are either primitive spectral types or low albedo, consistent with a cometary origin. So once again we have a consistent picture of candidate objects with primitive, possibly cometary, surface types.

Clearly, additional physical studies are required of the other objects listed in Tables 1 and 2 in order to gain a more complete picture of these extinct comet candidates. Although it may not be possible to conclusively identify these bodies as cometary using only Earth-based 
observations, such studies will help to identify the most probable candidates, which might then become the targets of spacecraft missions that will study them in far greater detail.

\section{Other Cometary Candidates}

Many asteroids have been suggested as extinct comet candidates over the past two decades, based on their physical or dynamical characteristics. In this Section we will examine some of these candidates in greater detail. To begin with, we consider the list of nine "probable" extinct comet candidates in Paper 1, listed in Table 3. In light of current data, how many of these would we still consider to be good candidates?

To begin with, 2060 Chiron, the first Centaur asteroid discovered, has since been found to display cometary activity (Meech and Belton 1993) and is clearly a comet, likely derived from the Kuiper belt or scattered disk. However, Bottke et al. (2002) find that only one of five NEOs in Table 3, 3553 Don Quixote, likely has a cometary origin. The other NEOs appear to be better explained, at least dynamically, as having come from the asteroid belt. One of these, 2201 Oljato, is spectral type Sq, suggesting a surface composition similar to ordinary chondrites. Although Oljato has been a popular candidate for an extinct cometary nucleus in the past (see Paper 1, also McFadden et al. 1993), both the dynamical and spectral data appear to rule it out.

Asteroid 944 Hidalgo, with $\mathrm{T}=2.067$, is in a dynamical regime that would argue very strongly for it being a cometary body, and equally likely for it being derived from the Kuiper belt, Oort cloud, or scattered disk. Asteroids 5201 Ferraz-Mello and 1984 BC are in dynamical regimes where they are possibly extinct JFCs, but without additional physical studies, it is difficult to say so for sure.

Asteroid 3200 Phaethon is an interesting case because it has been identified with the Geminid meteor shower (Whipple 1983; Williams and Wu 1993), one of the major regular showers each year. Our expectation is that meteor showers originate from comets; the Geminids is the only major shower definitively identified with an asteroid. Could 3200 Phaethon be an extinct comet nucleus? Its spectral type, $\mathrm{BF}$, is a primitive class, more likely to originate from the middle or outer asteroid belt than from the $v_{6}$ resonance, as suggested by Bottke et al. (2002). However, its albedo has been estimated as $0.11-0.17$, more typical of a non-primitive asteroid (Veeder et al. 1984; Green et al. 1985). Also, a source of F-type asteroids has been identified close to the 3:1 resonance in the Polana family in the main belt (Doressoundiram et al. 1998). As noted in Section 4, the dynamical integrations used by Bottke et al. to simulate the JFC evolution did not include the terrestrial planets or nongravitational forces, and this probably precluded them from producing objects in orbits like Comet $2 \mathrm{P} /$ Encke or even perhaps asteroid 3200 Phaethon. Searches for cometary activity in 3200 Phaethon have all been negative (Cochran and Barker 1984; Chamberlin et al. 1996). So we must conclude that the origin of this object remains an open question.

Two interesting objects are comets 107P/Wilson-Harrington and 133P/Elst-Pizarro, which are also catalogued as asteroids 4015 and 7968, respectively. In each case, these objects were briefly observed to display narrow, tail-like structures, which have been interpreted by 
some as cometary outbursts (Fernández et al. 1997; Boehnhardt et al. 1998). Lien (1998) has alternatively suggested that the structures were dust trails resulting from impact events. Both objects have Tisserand parameters greater than 3: 3.084 for P/Wilson-Harrington and 3.184 for $\mathrm{P} /$ Elst-Pizarro. In fact, these are the highest values of $\mathrm{T}$ for any objects classified as comets, with the exception of 95P/Chiron (which is not Jupiter-crossing). The orbit of Elst-Pizarro is particularly unusual as it is a typical low eccentricity, low inclination, main belt asteroid orbit (a $=3.158 \mathrm{AU}, \mathrm{e}=0.165, \mathrm{i}=1.38^{\circ}$ ). This orbit places it in the Themis collisional family. It is highly improbable that an extinct comet would evolve to such an orbit. A collision with other Themis family debris seems a more plausible explanation for 7968's transient activity.

In the case of Wilson-Harrington, the orbit is more typical of a JFC ( $\mathrm{a}=2.643 \mathrm{AU}, \mathrm{e}=$ $0.621, \mathrm{i}=2.78^{\circ}$ ) but Bottke et al. (2002) assign it only a $4 \%$ probability of having originated from the JFCs. They find the most probable source to be the outer main belt (65\%), though that result may again change if more complete integrations of the JFCs (including nongravitational forces) were to be included in their work. Wilson-Harrington's spectral type is CF, consistent with a cometary (or an outer main belt) origin, though as noted above, a source of F-type asteroids has also been identified close to the 3:1 resonance in the main belt (Doressoundiram et al. 1998). The case of Wilson-Harrington is problematic because its activity was observed on only one night, on photographic plates taken for the original Palomar Sky Survey in 1949, and the object is trailed on both images obtained (see Fernández et al. 1997); no activity was detected on plates taken three nights later. Subsequent searches for cometary activity in 4015 WilsonHarrington have all been negative (e.g., Chamberlin et al. 1996).

Some researchers have suggested possible links between weak meteoroid streams and Earth-crossing asteroids (Drummond 1982). For example, Kostolanský (1998) suggested possible stream identifications with 2102 Tantalus, 54961973 NA, and 1996 BT. However, Bottke et al. (2002) does not identify any of these as cometary candidates. Also, the possibility that these are extinct comets is generally not supported by taxonomic identifications, where they are available. Either the meteoroid stream identifications are coincidence or perhaps asteroids can be a source of meteoroid streams.

Yeomans (1991) searched for nongravitational motion in the orbits of 12 near-Earth asteroids and believed he had detected such evidence for 1566 Icarus and 1862 Apollo. However, he later showed (Yeomans 1992) that this was because of the failure to include relativistic terms in the motion of these objects. Since Icarus is spectral type Sq and Apollo is type Q, it would seem unlikely that these could be extinct comets.

\section{Discussion}

It now appears highly likely that there are a significant number of dormant or extinct cometary bodies among the asteroid population. We now have the tools and the understanding to begin identifying the most likely cometary candidates, and to follow up with telescopic physical studies that will help to confirm or refute those identifications. In addition, we have the promise of possible low-cost spacecraft missions that can study these objects in situ in far greater detail. 
The work of Bottke et al. (2002) allows us to identify likely cometary candidates among the NEO population. The most likely candidates, those with $\mathrm{P}_{\mathrm{JFC}}>50 \%$, all have Tisserand parameters $<3$. It is thus likely that additional asteroids with $\mathrm{T}<3$ but with $\mathrm{q}>1.3$ are also dormant or extinct comets. Potential improvements to the work of Bottke et al. will further refine our ability to identify cometary candidates through their dynamical properties. These improvements include better integrations of the Jupiter-family comet population where the terrestrial planets and nongravitational forces are included, improved integrations of the nearlyisotropic comet population, including, in particular the Halley-type comets, and improved statistics for the unbiased distribution of NEO orbits, based on increased discovery statistics and on data from some of the other ongoing NEO search programs.

Additional evidence for extinct comets comes from the discovery of objects in unusual orbits with large semi-major axes, eccentricities, and/or inclinations, and with $\mathrm{T}<2$. It is far more likely that these objects are derived from the Oort cloud and from objects in Halley-type orbits, than from the main asteroid belt. However, we must also remember that some small fraction of the Oort cloud is actually asteroidal bodies ejected there during the planetary zone clearing phase in the early solar system. Thus, these objects with $\mathrm{T}<2$ may still be asteroidal in nature, rather than cometary. Improved dynamical simulations of the ejection of these asteroids to the Oort cloud would be valuable in helping to understand this process.

The other key component is physical studies of the candidate comets, starting with those listed in Tables 1 and 2. The most important measurements are spectral identifications and albedo determinations, but rotation light curves, shape modeling, and other measurements will also provide useful clues to the true nature of the objects. Also, the same measurements need to be made of the known cometary nuclei so as to better determine the distributions of physical parameters for the progenitor population. Potential differences in these distributions, such as a lack of fast rotators among comets, will provide useful clues in determining the probability that a particular object has its origin as a comet or an asteroid. Radar studies may help to identify cometary surface types (Harmon et al. 1999), which appear to be low density and rough at scales from meters to kilometers.

It is also conceivable that progress in instrument technology as well as the increasing availability of very large aperture telescopes will allow us to identify weak cometary activity among the candidate comets, observations that so far have been unsuccessful.

Ultimately, some of the greatest gains will come from spacecraft missions, which will permit us to study active cometary nuclei and perhaps some extinct ones at close range. The recent success of Deep Space 1 in imaging the nucleus of P/Borrelly has been very valuable. Similarly valuable results can be expected from the Stardust mission now en route to 81P/Wild 2, and the CONTOUR and Deep Impact missions, to be launched in 2002 and 2004, respectively. However, the greatest results will come from ESA's Rosetta rendezvous and lander mission, to be launched in 2003, which will permit an in depth study of the nucleus of 46P/Wirtanen as it travels around the Sun. Since comets appear to be a very diverse population, additional comet rendezvous missions are highly desirable, in order to compare results and to provide criteria and clues for the detection of extinct nuclei among the asteroid population. 
Our understanding of both comets and asteroids has increased remarkably since the Asteroids II conference and book, 13 years ago. But at the same time we can only guess at how much further we will need to go to understand the complex interrelationships between these primordial bodies. There are many questions yet to be answered: How do comets physically evolve to dormant or extinct objects? What fraction of comets reach this end-state? Can comets become truly extinct or only temporarily dormant? What fraction of the Oort cloud population is asteroidal bodies? Can we find physical criteria that will definitively identify extinct cometary nuclei among the asteroid population, without the necessity for actually visiting them with spacecraft? It will be most interesting to see how much progress has been made in answering these questions at the Asteroids IV meeting.

\section{Acknowledgments}

We thank Mike Hicks, Rob Whiteley, Bobby Bus, and Rick Binzel for the use of their unpublished asteroid spectra, and Yan Fernández for his comet and asteroid albedos. We thank Don Yeomans, Alan Chamberlin, and especially Yan Fernández for useful comments on an earlier draft of this paper. This work was performed in part at the Jet Propulsion Laboratory and was funded in part by the NASA Origins and Planetary Geology and Geophysics Programs.

\section{References}

A’Hearn, M. (1988) Observations of cometary nuclei. Ann., Rev. Earth Planet. Sci. 16, 273-293.

Asphaug, E., and Benz, W. (1994) Density of comet Shoemaker-Levy-9 deduced by modeling breakup of the parent rubble pile. Nature 370, 120-121.

Asphaug, E., and Benz, W. (1996) Size, density, and structure of comet Shoemaker-Levy 9 inferred from the physics of tidal breakup. Icarus 121, 225-248.

Bailey, M. E. (1984) The steady-state 1/a distribution and the problem of cometary fading. MNRAS.211, 347-368.

Bailey, M. E., and Stagg, C. R. (1988) Cratering constraints on the inner Oort cloud: Steady-state models. MNRAS 235, 1-35.

Boehnhardt, H., Sekanina, Z., Fiedler, A., Rauer, H., Schulz, R., and Tozzi, G. (1998) Impact-induced activity of the asteroid-comet P/1996 N2 Elst-Pizarro: Yes or No? Highlights Astron. 11A, 233-237.

Brin, G. D., and Mendis, D. A. (1979) Dust release and mantle development in comets. Astrophys. J. 229, 402-408.

Bottke, W. F., Morbidelli, A., Jedicke, R., Petit, J.-M., Levison, H., Michel, P., and Metcalfe, T. S. (2002) Debiased orbital and absolute magnitude distributions of near-Earth objects. Icarus, in press.

Bowell, E., Hapke, B., Domingue, D., Lumme, K., Peltoniemi, J. and Harris, A. W. (1989) Application of photometric models to asteroids. In Asteroids II, eds. R. P. Binzel, T. Gehrels, and M. S. Matthews 
(Univ. Arizona Press, Tucson) pp. 524-556.

Carusi, A., Valsecchi, G. (1987) Dynamical evolution of short-period comets. In Interplanetary Matter, eds. Z. Ceplecha and P. Pecina (Czech. Acad. Sci., Ondrejov), pp. 21-28.

Chamberlin, A. B., McFadden, L., Schulz, R., Schleicher, D. G.,00 and Bus, S. J. (1996) 4015

Wilson-Harrington, 2201 Oljato, and 3200 Phaethon: Search for CN Emission. Icarus 119, 173-181

Cochran, A. L., and Barker, E. S. (1984) Minor planet 1983 TB: A dead comet? Icarus 59, 296-300.

Degewij, J., and Tedesco, E. F. (1982) Do comets evolve into asteroids? Evidence from physical studies. In Comets, ed. L. L. Wilkening (Univ. Arizona Press, Tucson), pp. 665-695.

Dones, L., Levison, H., Duncan, M., and Weissman, P. (2000) Formation of the Oort cloud revisited. Bull. Amer. Astron. Soc. 32, 1060 (abstract).

Donn, B., and Hughes D. (1986) A fractal model of a cometary nucleus formed by random accretion. In $20^{\text {th }}$ ESLAB Symposium on the Exploration of Halley's Comet, eds. B. Battrick, E. J. Rolfe, and R.

Reinhard, ESA SP-250, 3, 523-524.

Doressoundiram, A., Cellino, A., di Martino, M., Migliorini, F. and Zappalà, V. (1998) The puzzling case of the Nysa-Polana family finally solved? Bull. Amer. Astron. Soc. 30, 505 (abstract).

Drummond, J. D. (1982) Theoretical meteor radiants of Apollo, Amor, and Aten asteroids. Icarus 49, 143-153.

Duncan, M., Quinn, T., and Tremaine, S. (1987) The formation and extent of the solar system comet cloud. Astron. J. 94, 1330-1338.

Duncan, M., Quinn, T., and Tremaine, S. (1988) The origin of short-period comets. Astrophys. J. 328, L69-L73.

Duncan, M. J., and Levison, H. F. (1997) A scattered comet disk and the origin of Jupiter family comets. Science 276, 1670-1672.

Eggers, S. (1999) Cometary dynamics during the formation of the solar system. Ph.D. Thesis, Max-Planck-Institüt für Aeronomie.

Everhart, E. (1977) The evolution of comet orbits as perturbed by Uranus and Neptune. In Comets, Asteroids, Meteorites: Interrelations, Evolution and Origins, ed. A. H. Delsemme, (Univ. Toledo Press, Toledo), pp. 99-104.

Fanale, F. P., and Salvail, J. R. (1984) An idealized short period comet model: surface insolation, $\mathrm{H}_{2} \mathrm{O}$ flux, dust flux and mantle development. Icarus 60, 476-511.

Fernández, J. A. (1980) On the existence of a comet belt beyond Neptune. MNRAS 192, 481-491.

Fernández, J. A. (1997) The formation of the Oort cloud and the primitive galactic environment. Icarus 129, 106-119. 
Fernández, J. A., and Ip, W.-H. (1991) Statistical and evolutionary aspects of cometary orbits. In Comets in the Post-Halley Era, eds. R. L. Newburn Jr., M. Neugebauer, and J. Rahe (Kluwer, Dordrecht), pp. 487-536.

Fernández, J. A., Rickman, H., and Kamél, L. (1992) The population size and distribution of perihelion distances of the Jupiter family. In Periodic Comets, eds. J. A. Fernandez and H. Rickman (Univ.

Republica, Montevideo) pp. 143-157).

Fernández, J. A., Tancredi, G., Rickman, H., and Licandro, J. (1999) The population, magnitudes, and sizes of Jupiter family comets. Astron \& Astrophys. 352, 327-340.

Fernández, J. A. and Brunini, A. (2000) The buildup of a tightly bound comet cloud around an early Sun immersed in a dense Galactic environment: Numerical experiments. Icarus 145, 580-590

Fernández, Y. R., McFadden, L. A., Lisse, C. M., Helin, E. F., and Chamberlin, A. B. (1997) Analysis of POSS images of comet-asteroid 107P/1949 W1 Wilson Harrington. Icarus 128, 114-126.

Fernández, Y. R. (1999) Physical properties of cometary nuclei. Ph.D. thesis, University of Maryland.

Fernández, Y. R., Jewitt, D. C., and Shephard, S. S. (2001) Low albedos among extinct comet candidates. Astrophys. J. 553, L197-L200.

Green, S. F., Meadows, A. J., and Davies, J. K. (1985) Infrared observations of the extinct cometary candidate minor planet (3200) 1983 TB. MNRAS 214, 29-36.

Harmon, J. K., Campbell, D. B., Ostro, S. J., and Nolan, M. C. (1999) Radar observations of comets. Plan. \& Spa. Sci. 47, 1409-1422.

Harris, N. W., and Bailey, M. E. (1998) Dynamical evolution of cometary asteroids. MNRAS 297, 12271236.

Hicks, M. D., Buratti, B. J., Newburn, R. L., and Rabinowitz, D. L. (2000) Physical observations of 1996 PW and 1997 SE5: Extinct comets or D-type asteroids? Icarus 143, 354-359.

Hills, J. G. (1981) Comet showers and the steady-state infall of comets from the Oort cloud. Astron. J. 86, 1730-1740.

Jewitt, D., and Luu, J. (1993) Discovery of the candidate Kuiper belt object 1992 QB $_{1}$. Nature 362, 730-732.

Johnson, R. E., Cooper, J. F., Lanzerotti, L. J., and Strazzula, G. (1987) Radiation formation of a nonvolatile comet crust. Astron. Astrophys. 187, 889-892.

Kamél, L. (1991) The evolution of P/Encke's light curve - No secular fading, a vanishing perihelion asymmetry. Icarus 93, 226-245.

Keller, H. U., Arpigny, C., Barbieri, C., Bonnett, R. M., Cazes, S., Coradini, M., Cosmovici, C. B., Curdt, W., Delamere, W. A., Huebner, W. F., Hughes, D. W., Jamar, C., Kramm, R., Malaise, D., Reitsema, H., Schmidt, H. U., Schmidt, K., Schmidt, W. K. H., Seige, P., Whipple, F. L., and Wilhelm, K. (1986) Observations by the Halley multicolour camera. In 20th ESLAB Symposium on the Exploration of 
Halley's Comet, eds. B. Battrick, E. J. Rolfe, and R. Reinhard, ESA SP-250, 2, 347-350.

Kostolanský, E. (1998) On asteroidal meteoroid streams detection. Contrib. Astron. Obs. Skalnaté Pleso 28, 22-30.

Kresák, L. (1979) Dynamical interrelations among comets and asteroids. In Asteroids, ed. T. Gehrels (Univ. Arizona Press, Tucson), pp. 289-309.

Kresák, L. (1987) Dormant phases in the aging of periodic comets. Astron. Astrophys. 187, 906-908.

Kronk, G. W. (1984) Comets, A Descriptive Catalog. (Enslow Publ., Hillside, NJ), 331 pp.

Kuiper, G. P. (1951) On the origin of the solar system. In Astrophysics, ed. J. A. Hynek (McGraw Hill, New York), pp. 357-424.

Levison, H. F. (1996) Comet taxonomy. In Completing the Inventory of the Solar System, eds. T. W. Rettig and J. M. Hahn, ASP Conf. Ser. 107, pp. 173-191.

Levison, H. F., and Duncan, M. J. (1994) The long-term dynamical behavior of short-period comets. Icarus 108, 18-36.

Levison, H. F., and Duncan, M. J. (1997) From the Kuiper belt to Jupiter-family comets: The spatial distribution of ecliptic comets. Icarus 127, 13-32.

Levison, H. F., Duncan, M. J., Zahnle, K., Holman, M., and Dones, L. (2000) Planetary impact rates from ecliptic comets. Icarus 143, 415-420.

Levison, H. F., Dones, L., and Duncan, M. J. (2001) The origin of Halley-type comets: Probing the inner Oort cloud. Astron. J. 121, 2253-2267.

Lien, D. (1998) Asteroid debris trails: evidence for recent collisions in the asteroid belt. Bull. Amer. Astron. Soc. 30, 1035 (abstract).

Lowry, S. C. (2001) Physical properties of cometary nuclei and studies of distant activity. Ph.D. Thesis, Queens University, Belfast.

Luu, J. X. (1993) Spectral diversity among the nuclei of comets. Icarus 104, 138-148.

Malhotra, R., Duncan, M. J., and Levison, H. F. (2000) Dynamics of the Kuiper belt. In Protostars and Planets IV, eds. V. Mannings, A. P. Boss, and S. S. Russell (Univ. Arizona Press, Tucson), pp. 12311254.

Marsden, B. G., Sekanina, Z., and Everhart, E. (1978) New osculating orbits for 110 comets and the analysis of the original orbits of 200 comets. Astron. J. 83, 64-71.

Marsden, B. G., and Williams, G. V. (1999) Catalogue of Cometary Orbits 1999. Minor Planet Center, $127 \mathrm{pp}$.

McFadden, L. A., Cochran, A. L., Barker, E. S., Cruikshank, D. P., and Hartmann, W. K. (1993) The enigmatic object 2201 Oljato - Is it an asteroid or an evolved comet? J. Geophys. Res. 98, 3031-3041. 
Meech, K. J., and Belton, M. J. S. (1989) 2060 Chiron. IAUC Circ. 4770.

Meech, K. J. (in press) Physical properties of comets. In Asteroids, Comets, Meteors 1996, ed. A.-C. Levasseur-Regourd (Kluwer, Dordrecht).

Moore, M. H., Donn, B., Khanna, R., and A'Hearn, M. F. (1983) Studies of proton-irradiated cometarytype ice mixtures. Icarus 54, 388-405.

Newton, H. A. (1891) Capture of comets by planets. Astron. J. 11, 73-75.

Newton, H. A. (1893) On the capture of comets by planets, especially their capture by Jupiter. Mem. Natl. Acad. Sci. 6, 7-23.

Oort, J. H. (1950) The structure of the cloud of comets surrounding the solar system and a hypothesis concerning its origin. Bull. Astron. Inst. Neth. 11, 91-110.

Peale, S. J. (1989) On the density of Halley's comet. Icarus 82, 36-49.

Prialnik, D., and Bar-Nun, A. (1988) The formation of a permanent dust mantle and its effect on cometary activity. Icarus $\mathbf{7 4 ,}$, 272-283.

Quinn, T., Tremaine, S., and Duncan, M. (1990) Planetary perturbations and the origin of short-period comets. Astrophys. J. 355, 667-679.

Rickman, H. (1986) Masses and densities of comets Halley and Kopff. In The Comet Nucleus Sample Return Mission, ESA SP-249, pp. 195-205.

Sekanina, Z. (1982) The problem of split comets in review. In Comets, ed. L. L. Wilkening (Univ. Arizona Press, Tucson), pp. 251-287.

Sekanina, Z. (1991) Outgassing asymmetry of periodic Comet Encke. II - Apparitions 1868-1918 and a study of the nucleus evolution. Astron. J. 96, 1455-1475.

Scotti, J. V. (1996) Near-Earth object surveying in the late $20^{\text {th }}$ century. In Completing the Inventory of the Solar System, eds. T. W. Rettig and J. M. Hahn, ASP Conf. Ser. 107, 107-113.

Shoemaker, E. M., and Wolfe, R. F. (1982) Cratering time scales for the Galilean satellites. In Satellites of Jupiter, ed. D. Morrison (Univ. Arizona Press, Tucson), pp. 277-339

Stephenson, F. R., Yau, K. K. C., and Hunger H. (1985) Records of Halley's comet on Babylonian tablets. Nature 314, 587-592.

Veeder, G. J., Kowal, C., and Matson, D. L. (1984) The Earth-Crossing Asteroid 1983 TB. Lun. \& Planet. Sci Conf. 15, 878-879 (abstract).

Weaver, H. A., Sekanina, Z., Toth, I., Delahodde, C. E., Hainaut, O. R., Lamy, P. L., Bauer, J. M., A'Hearn, M. F., Arpigny, C., Combi, M. R., Davies, J. K., Feldman, P. D., Festou, M. C., Hook, R., Jorda, L., Keesey, M. S. W. Lisse, C. M., Marsden, B. G., Meech, K. J., Tozzi, G. P., and West, R. (2001) HST and VLT investigations of the fragments of comet C/1999 S4 (LINEAR). Science 292, 1329-1334. 
Weissman, P. R. (1979) Physical and dynamical evolution of long-period comets. In Dynamics of the Solar System, ed. R. L. Duncombe (D. Reidel, Dordrecht), pp. 277-282.

Weissman, P. R. (1980) Physical loss of long-period comets. Astron. \& Astrophys. 85, 191-196.

Weissman, P. R. (1985) Dynamical evolution of the Oort cloud. In Dynamics of Comets: Their Origin and Evolution, eds. A. Carusi and G. D. Valsecchi (D. Reidel, Dordrecht), pp. 87-96.

Weissman, P. R. (1986) Are cometary nuclei primordial rubble piles? Nature 320, 242-244.

Weissman, P. R. (1987) Post-perihelion brightening of Halley's Comet: Spring time for Halley. Astron. \& Astrophys, 187, 873-878.

Weissman, P. R. (1990) The cometary impactor flux at the Earth. In Global Catastrophes in Earth History, eds. V. L. Sharpton and P. D. Ward, Geol. Soc. Amer. Special Paper 247, pp. 171-180.

Weissman, P. R. (1993) No, we are not in a cometary shower. Bull. Amer. Astron. Soc. 25, 1063 (abstract).

Weissman, P. R. (1996a) The Oort cloud. In Completing the Inventory of the Solar System, eds. T. W. Rettig and J. M. Hahn, ASP Conf. Ser. 107, 265-288.

Weissman, P. R. (1996b) Star passages through the Oort cloud. Earth, Moon \& Planets 72, 25-30.

Weissman, P. R., A'Hearn, M. F., McFadden, L. A., and Rickman, H. (1989) Evolution of comets into asteroids. In Asteroids II, eds. R. P. Binzel, T. Gehrels, and M. S. Matthews (Univ. Arizona Press, Tucson), pp. 880-920.

Weissman, P. R., and Levison, H. F. (1997) Origin and evolution of the unusual object 1996 PW: Asteroids from the Oort cloud? Astrophys. J. 488, L133-136.

Weissman, P. R., and Stern, S. A. (1998) Physical processing of cometary nuclei. In Workshop on Analysis of Returned Comet Nucleus Samples, ed. S. Chang, NASA Conf. Pub. 10152, pp. 119-166.

Weissman, P. R., and Lowry, S. C. (2001) The size distribution of cometary nuclei. Bull. Amer. Astron. Soc. 33, 1094 (abstract).

Wetherill, G. W. (1994) Possible consequences of absence of Jupiters in planetary systems. Astrophys. \& Spa. Sci. 212, 23-32.

Wetherill, G. W. (1988) Where do the Apollo objects come from? Icarus 76, 1-18.

Wetherill, G. W. (1991) End products of cometary evolution: Cometary origin of Earth-crossing bodies of asteroidal appearance. In Comets in the Post-Halley Era, eds. R. L. Newburn Jr., M. Neugebauer, and J. Rahe (Kluwer, Dordrecht), pp. 537-556.

Whipple, F. L. (1950) A comet model. I. The acceleration of comet Encke. Astrophys. J. 111, 375-394.

Whipple, F. L. (1962) On the distribution of sernimajor axes among comet orbits. Astron. J. 67, 1-9. 
Whipple, F. L. (1964) Evidence for a comet belt beyond Neptune. Proc. Natl. Acad. Sci. U.S. 51, 711718.

Whipple, F. L. (1983) 1983 TB and the Geminid meteors. IAU Circ. 3881.

Wiegert, P., and Tremaine S. (1999) The evolution of long-period comets. Icarus 137, 84-121.

Williams, I. P., and Wu, Z. (1993) The Geminid meteor stream and asteroid 3200 Phaethon. MNRAS 262, 231-248.

Yau, K., Yeomans, D., and Weissman, P. (1994) The past and future motion of comet Swift-Tuttle. MNRAS 266, 305-316.

Yeomans, D. K. (1991) A comet among the near-Earth asteroids? Astron. J. 101, 1920-1928.

Yeomans, D. K. (1992) Erratum: A comet among the near-Earth asteroids? Astron. J. 104, 1266.

Yeomans, D. K., Yau, K., and Weissman, P. R. (1996) The upcoming approach of periodic comet Tempel-Tuttle and the Leonid meteors. Icarus 124, 407-413.

Zahnle, K., Dones, L., and Levison, H. F. (1998) Cratering rates on the Galilean satellites. Icarus 136, 202-222. 


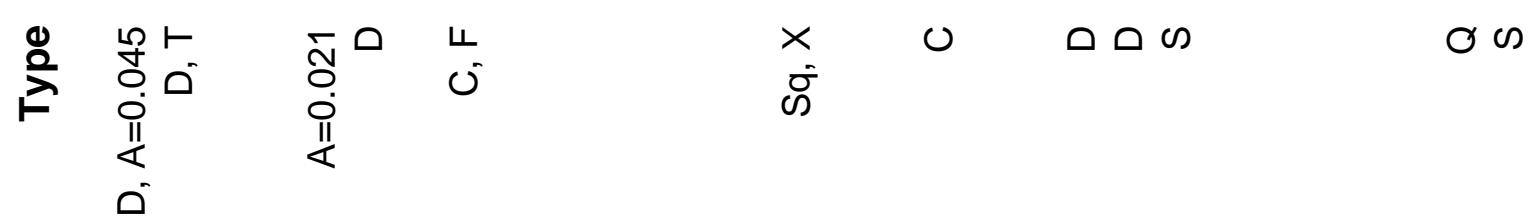

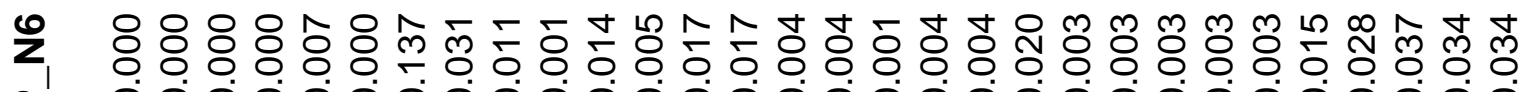

Q

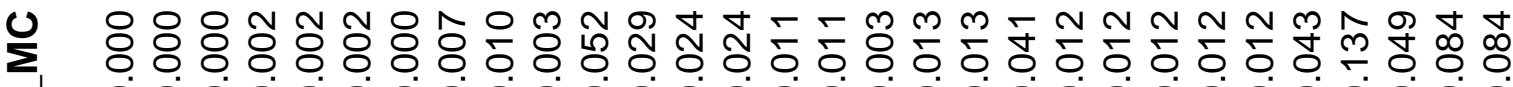

Q

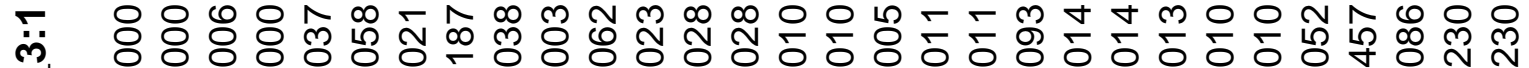

a

m

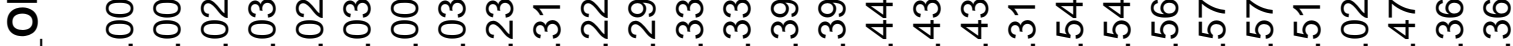

a 000000000000000000000000000000

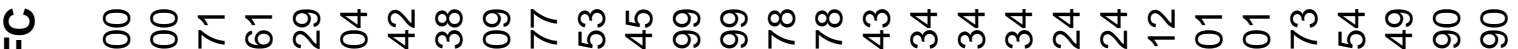

㭊 $\quad$ ।

0

- $\quad \forall \varphi \overline{0}$ m

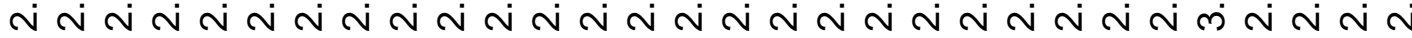

一 क 0 N

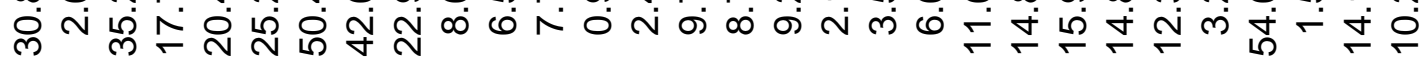

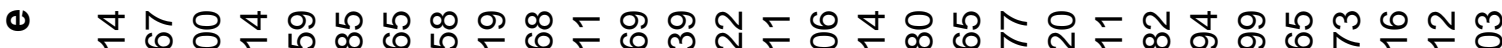
К

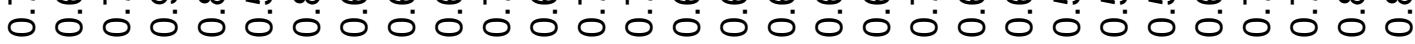

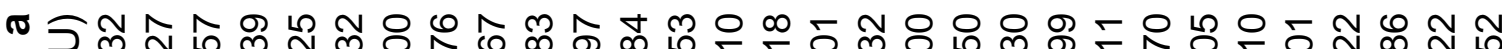

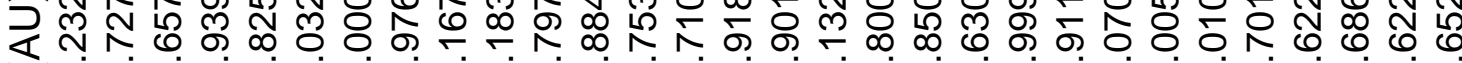
$\checkmark$ m

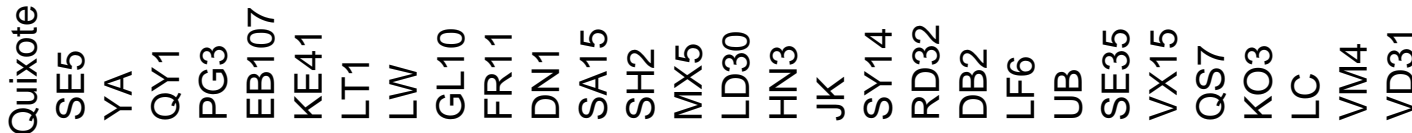

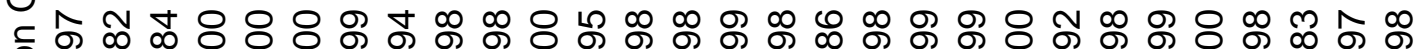

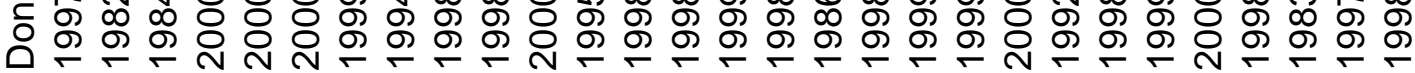
กิ 


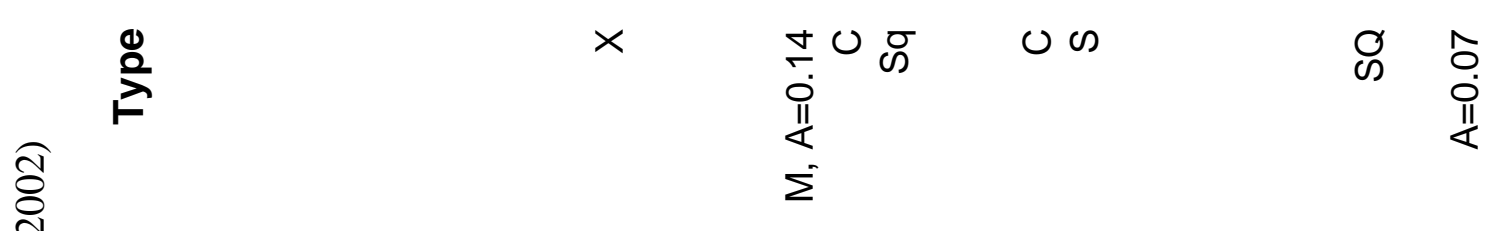

는

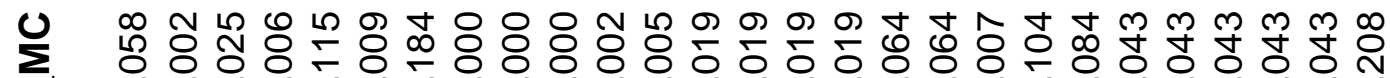

a

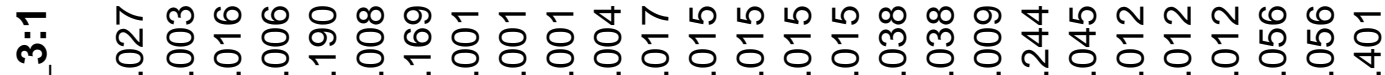

a

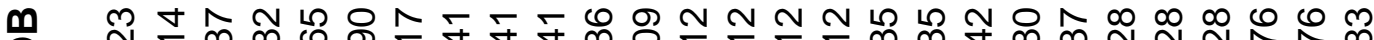

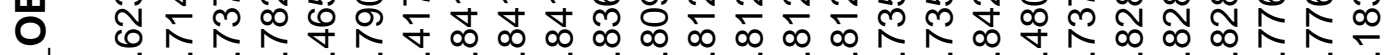

ـ ا

U.

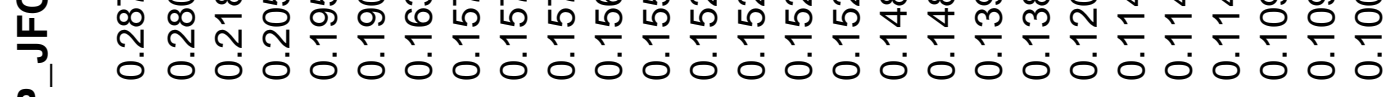

ฉ

बㅅN ヘ $\sim$ ヘ

一 ○ ウ

- ㄱํㅇํํ

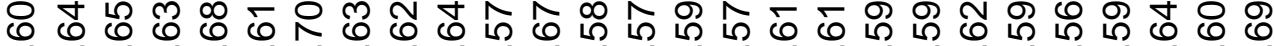

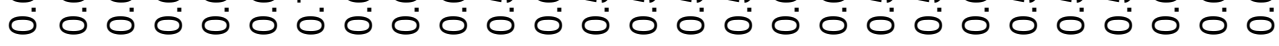

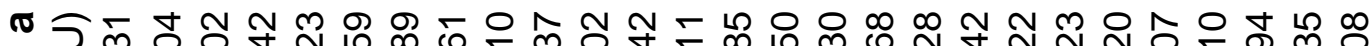
飞 -

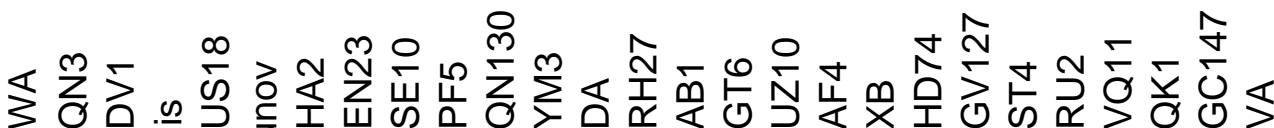

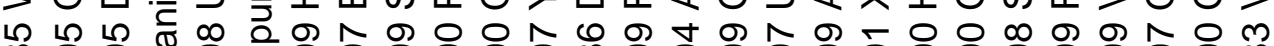

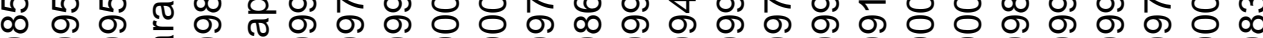

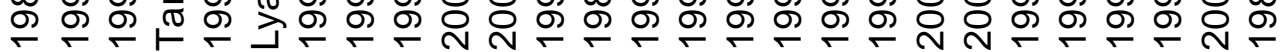

\begin{tabular}{|c|c|c|}
\hline ભ̊ & స్ & 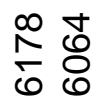 \\
\hline
\end{tabular}


Table 2. Asteroids in Comet-Like Orbits with Tisserand Parameter $<2$

\begin{tabular}{|c|c|c|c|c|c|c|c|c|c|}
\hline Number & Name & $\begin{array}{c}a \\
(A U)\end{array}$ & e & i & H & $\mathbf{T}$ & $\stackrel{q}{(A U)}$ & $\begin{array}{c}P \\
\text { (yrs) }\end{array}$ & $\begin{array}{l}\text { Spectral } \\
\text { Type }\end{array}$ \\
\hline 5335 & Damocles & 11.818 & 0.8667 & 62.047 & 13.3 & 1.145 & 1.575 & 40.63 & $\mathrm{D}$ \\
\hline 15504 & 1998 RG33 & 9.433 & 0.7726 & 34.936 & 12.1 & 1.953 & 2.145 & 28.97 & \\
\hline \multirow[t]{9}{*}{20461} & 1999 LD31 & 24.429 & 0.9025 & 160.213 & 13.8 & -1.543 & 2.381 & 120.74 & \\
\hline & 1996 PW & 305.934 & 0.9917 & 29.782 & 14.0 & 1.731 & 2.547 & 5351.10 & $\mathrm{D}$ \\
\hline & 1997 MD10 & 26.865 & 0.9427 & 59.047 & 16.0 & 0.974 & 1.540 & 139.25 & \\
\hline & 1998 WU24 & 15.207 & 0.9069 & 42.564 & 15.0 & 1.403 & 1.415 & 59.30 & \\
\hline & 1999 LE31 & 8.155 & 0.4718 & 151.879 & 12.4 & -1.309 & 4.307 & 23.29 & $A=0.031$ \\
\hline & 1999 XS35 & 18.044 & 0.9474 & 19.473 & 17.2 & 1.412 & 0.949 & 76.65 & \\
\hline & 2000 AB229 & 52.163 & 0.9561 & 68.715 & 14.0 & 0.773 & 2.290 & 376.74 & \\
\hline & 2000 DG8 & 10.773 & 0.7930 & 129.426 & 13.1 & -0.630 & 2.230 & 35.36 & $A=0.027$ \\
\hline & $2000 \mathrm{HE} 46$ & 24.191 & 0.9029 & 158.340 & 14.6 & -1.507 & 2.349 & 118.98 & $A=0.023$ \\
\hline
\end{tabular}

Table 3. Probable Extinct Comet Candidates from Weissman et al. (1989)

\begin{tabular}{|c|c|c|c|c|c|c|c|}
\hline Number & Name & $\begin{array}{c}a \\
(A U)\end{array}$ & e & i & $\mathbf{T}$ & $\begin{array}{l}\text { Spectral } \\
\text { Type }\end{array}$ & $\begin{array}{l}\text { Likely Source Region } \\
\text { (Bottke et al. 2002) }\end{array}$ \\
\hline 944 & Hidalgo & 5.752 & 0.661 & 42.557 & 2.067 & $\mathrm{D}$ & \\
\hline 2060 & Chiron & 13.602 & 0.380 & 6.940 & 3.352 & $B$ & \\
\hline 2101 & Adonis & 1.874 & 0.765 & 1.349 & 3.550 & & $v_{6} 43 \% ; \quad$ OB $32 \%$ \\
\hline 2201 & Oljato & 2.171 & 0.713 & 2.517 & 3.302 & $\mathrm{Sq}$ & $\mathrm{v}_{6} 55 \% ; \quad$ MC $27 \%$ \\
\hline 2212 & Hephaistos & 2.166 & 0.834 & 11.769 & 3.100 & & $\mathrm{v}_{6} 44 \% ; \quad$ MC $38 \%$ \\
\hline 3200 & Phaethon & 1.271 & 0.890 & 22.166 & 4.512 & $\mathrm{BF}$ & $v_{6} 52 \% ;$ MC 24\%; 3:1 24\% \\
\hline 3552 & Don Quixote & 4.232 & 0.714 & 30.816 & 2.314 & $\mathrm{D}$ & JFC $100 \%$ \\
\hline \multirow[t]{2}{*}{5201} & Ferraz-Mello & 3.173 & 0.518 & 4.053 & 2.973 & & \\
\hline & 1984 BC & 3.493 & 0.534 & 21.364 & 2.780 & & \\
\hline
\end{tabular}




\section{Figure Captions}

Figure 1. Hubble Space Telescope image of Comet LINEAR, D/1999 S4, on August 5, 2000, showing fragments of the disintegrating nucleus. This long-period comet disrupted close to perihelion at 0.765 AU from the Sun on July 26, 2000.

Figure 2. Composite image of the nucleus of Comet Halley (left), taken by the Giotto spacecraft on March 14, 1986, and the nucleus of Comet Borrelly (right) taken by the Deep Space 1 spacecraft on September 23, 2001. The Sun is at the left in both images. The Giotto spacecraft approached the nucleus from the dark side at a phase angle of $\sim 107^{\circ}$. Several distinct jets of gas and dust are seen emanating from the dayside of the nucleus. The entire Halley nucleus shape is silhouetted against the bright dust coma. The nucleus is $\sim 16 \times 8 \times 8 \mathrm{~km}$ in diameter $(\mathrm{CH}$. U. Keller, Max Planck Institut fur Aeronomie). The Borrelly image, taken at a phase angle of $\sim 52^{\circ}$, shows a dark, irregular nucleus $\sim 8$ $\mathrm{x} 4 \mathrm{x} 4 \mathrm{~km}$ with a highly irregular surface topography and active jets (not visible in this version of the image) originating near the center of the nucleus.

Figure 3. Location of all known comets and asteroids ( $\mathrm{H}$ brighter than 18) on an (a,e) scatter diagram of the inner solar system. Comets are shown by open circles, asteroids by filled circles. Solid lines show the boundaries of the region where objects are Earth-crossing $(\mathrm{q}<1.017$ AU and $\mathrm{Q}>0.983$ $\mathrm{AU}$ ). The dot-dash line is for $\mathrm{q}=1.3 \mathrm{AU}$, which defines the Amor-type (Earth-approaching) objects. Dashed lines show $\mathrm{T}=3$, the traditional boundary between cometary and asteroidal orbits; the dotted line shows $\mathrm{T}=2$, the boundary between ecliptic and nearly-isotropic comets. Data taken from the Minor Planet Center orbit files for comets and asteroids, March 8, 2001.

Figure 4. The cumulative inclination distribution of Jupiter-family comets with perihelion distances less than 2.5 AU. The bold solid curve is the observed distribution taken from Marsden and Williams' (1999) catalog. The top dotted curve shows the cumulative inclination distribution of LD97's simulated comets when they were first injected into the JFC region with $q<2.5 \mathrm{AU}$. The bottom dotted curve shows the inclination distribution that LD97 predicts if the JFCs are in steady-state equilibrium and if they remain active throughout their time in the JFC region. The middle dotted curve presents the distribution of LD97's best fit model with $t_{e}=12,000$ years.

Figure 5. Location on the (a,e) diagram of the 57 extinct comet candidates with $\mathrm{P}_{\mathrm{JFC}}>10 \%$ from Bottke et al. (2002). Twenty candidates with $\mathrm{P}_{\mathrm{JFC}}>50 \%$ are shown by open circles; 37 candidates with $50 \%>\mathrm{P}_{\mathrm{JFC}}>10 \%$ are shown by filled circles. Labeled curves are the same as in Figure 3.

Figure 6. The cumulative inclination distribution of observed comets with perihelia within 1.3 AU of the Sun taken from Marsden and Williams' (1999) catalog. The solid, dashed, and dotted curves are the JFCs, HTCs, and LPCs, respectively. We only include LPCs with a "quality code" of 1A or 1B. A random distribution would be a diagonal line from the lower left to the upper right in this figure. 


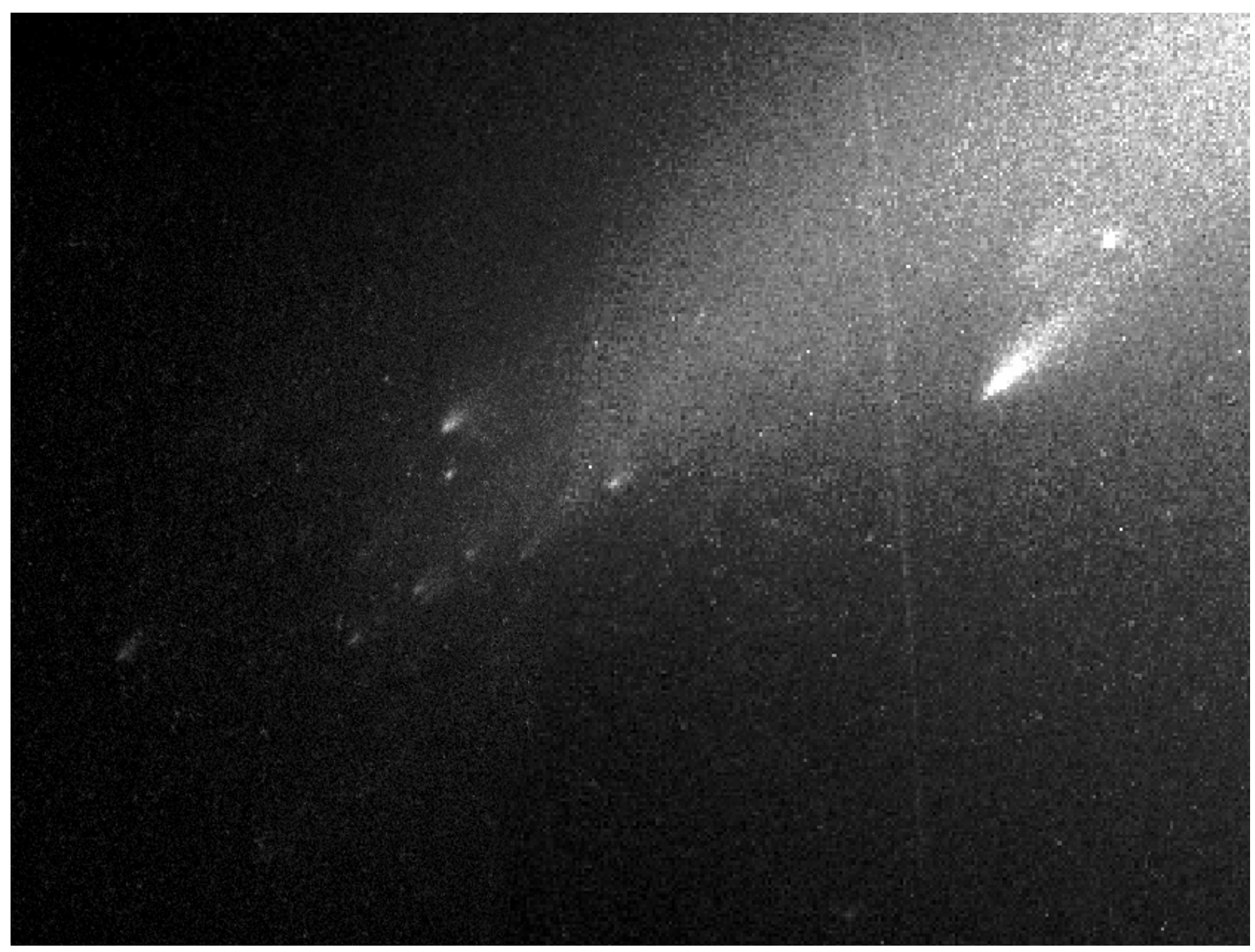

Figure 1. 

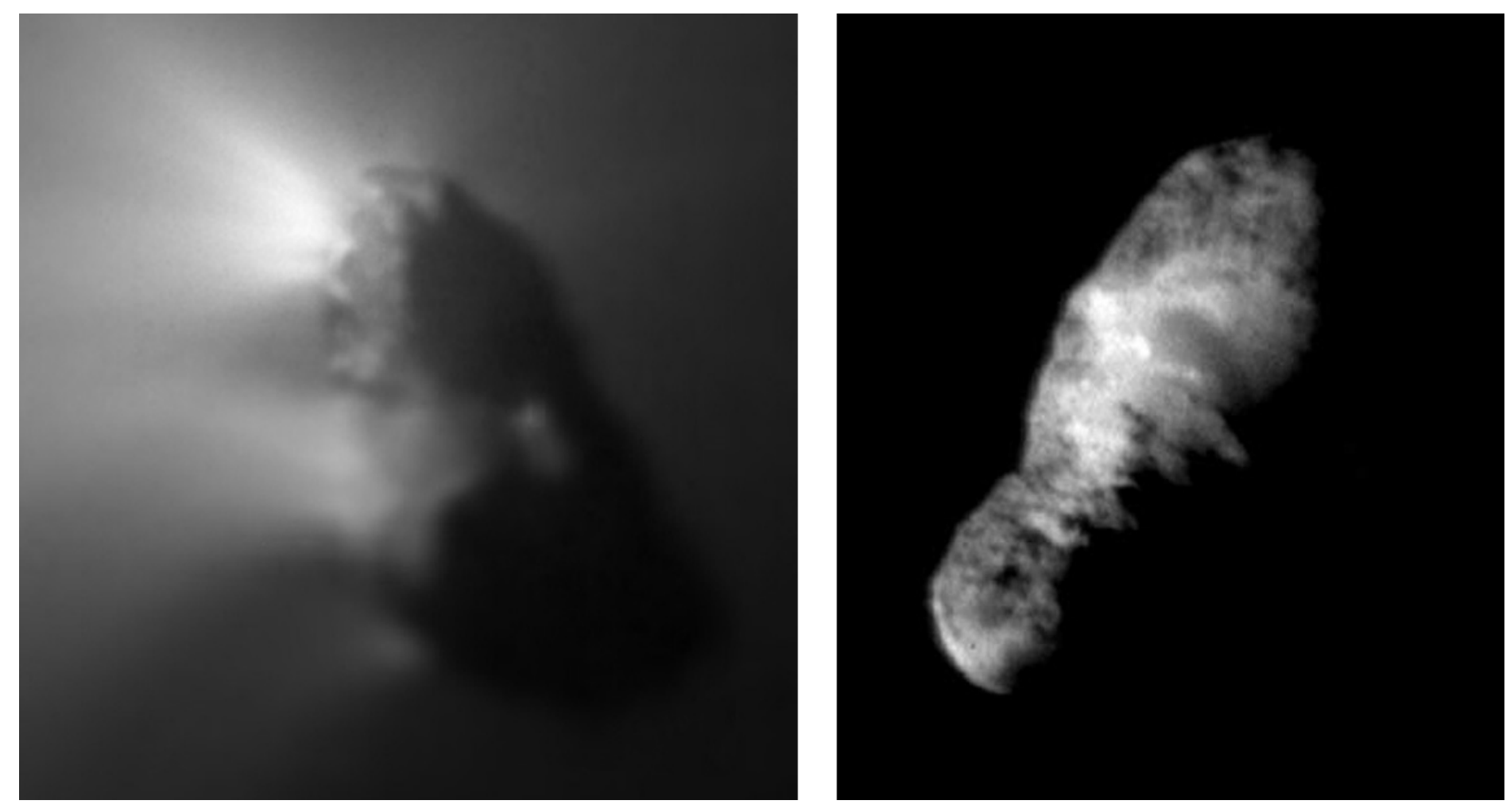

Figure 2. 


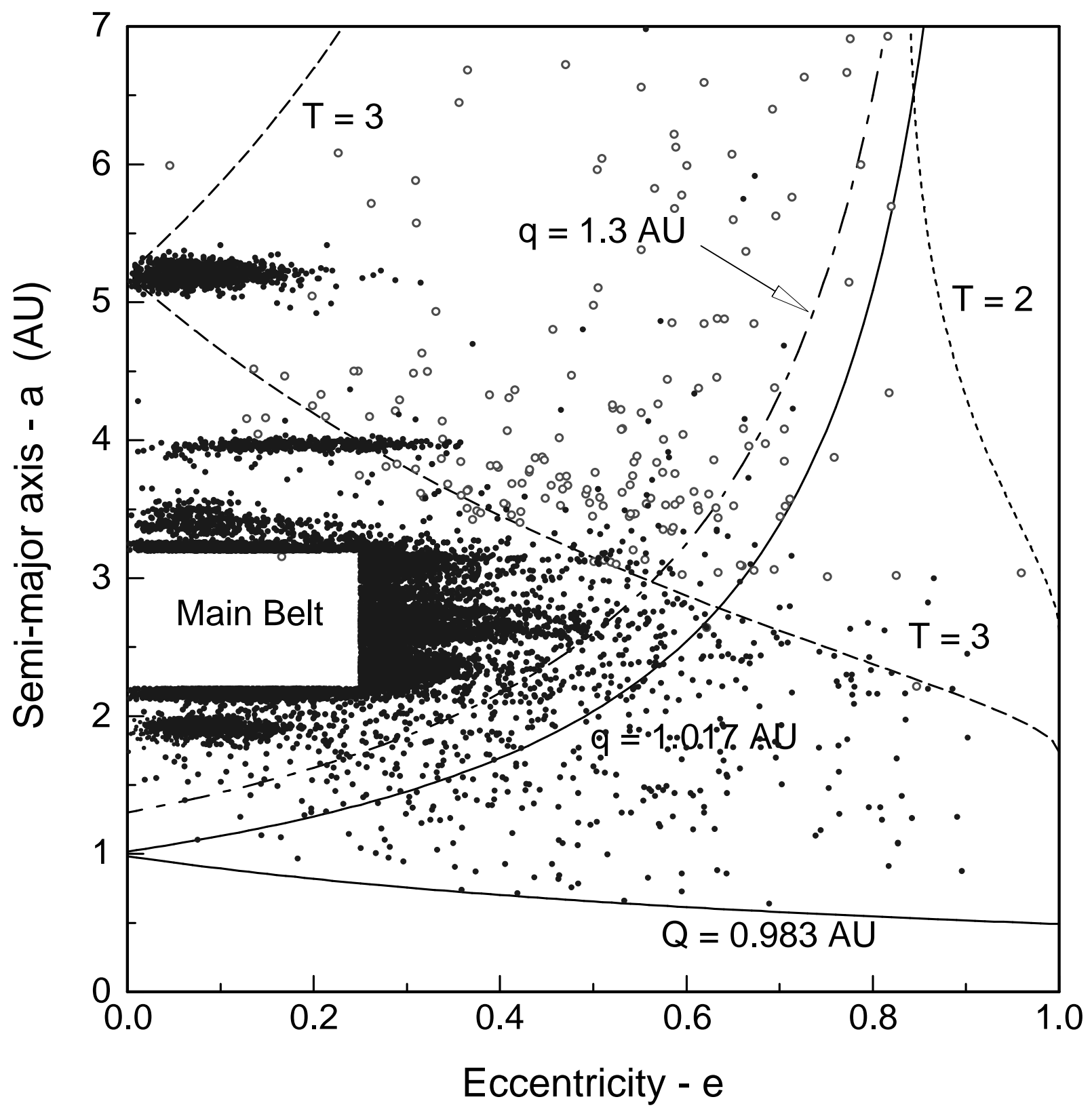

Figure 3. 


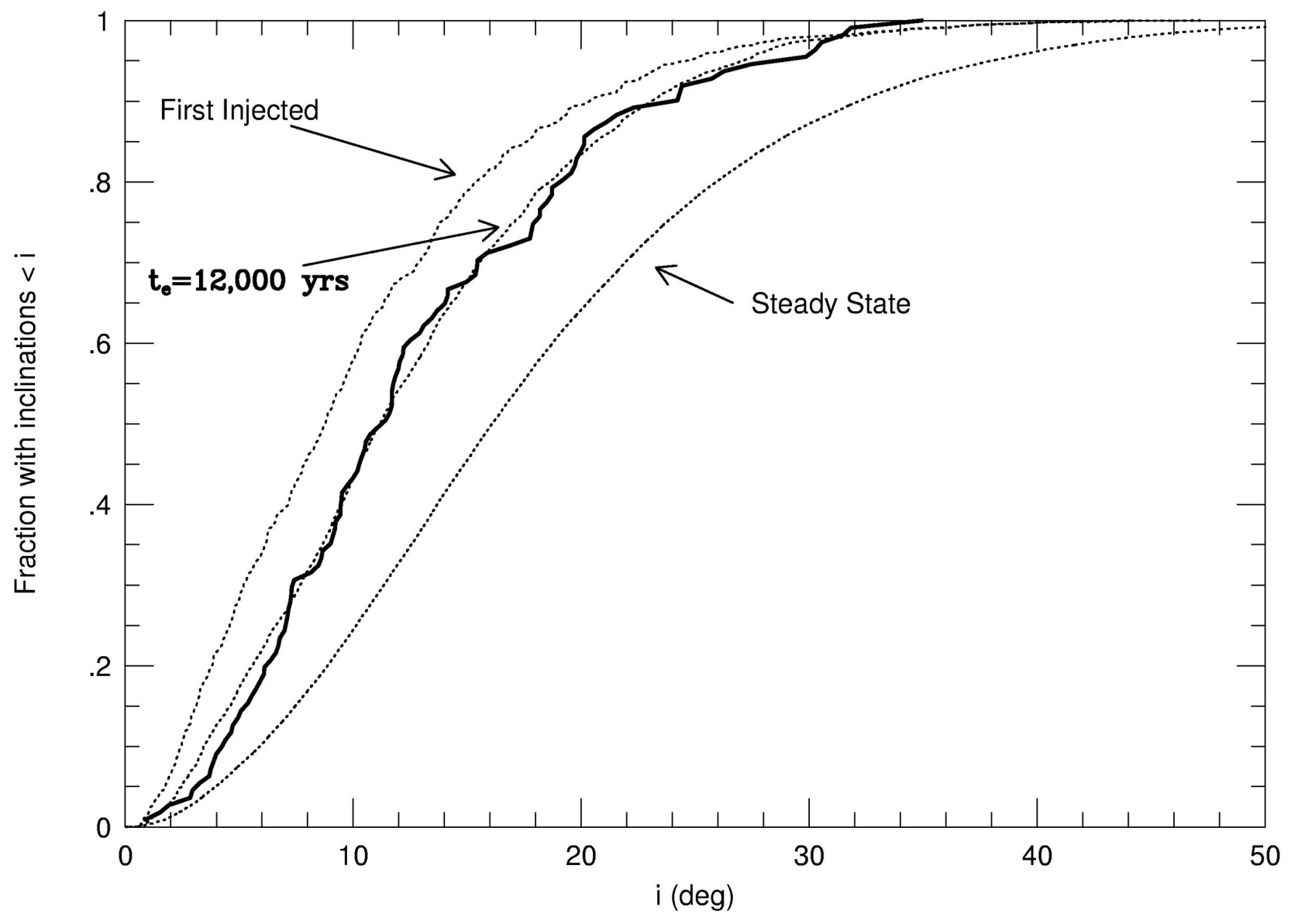

Figure 4. 


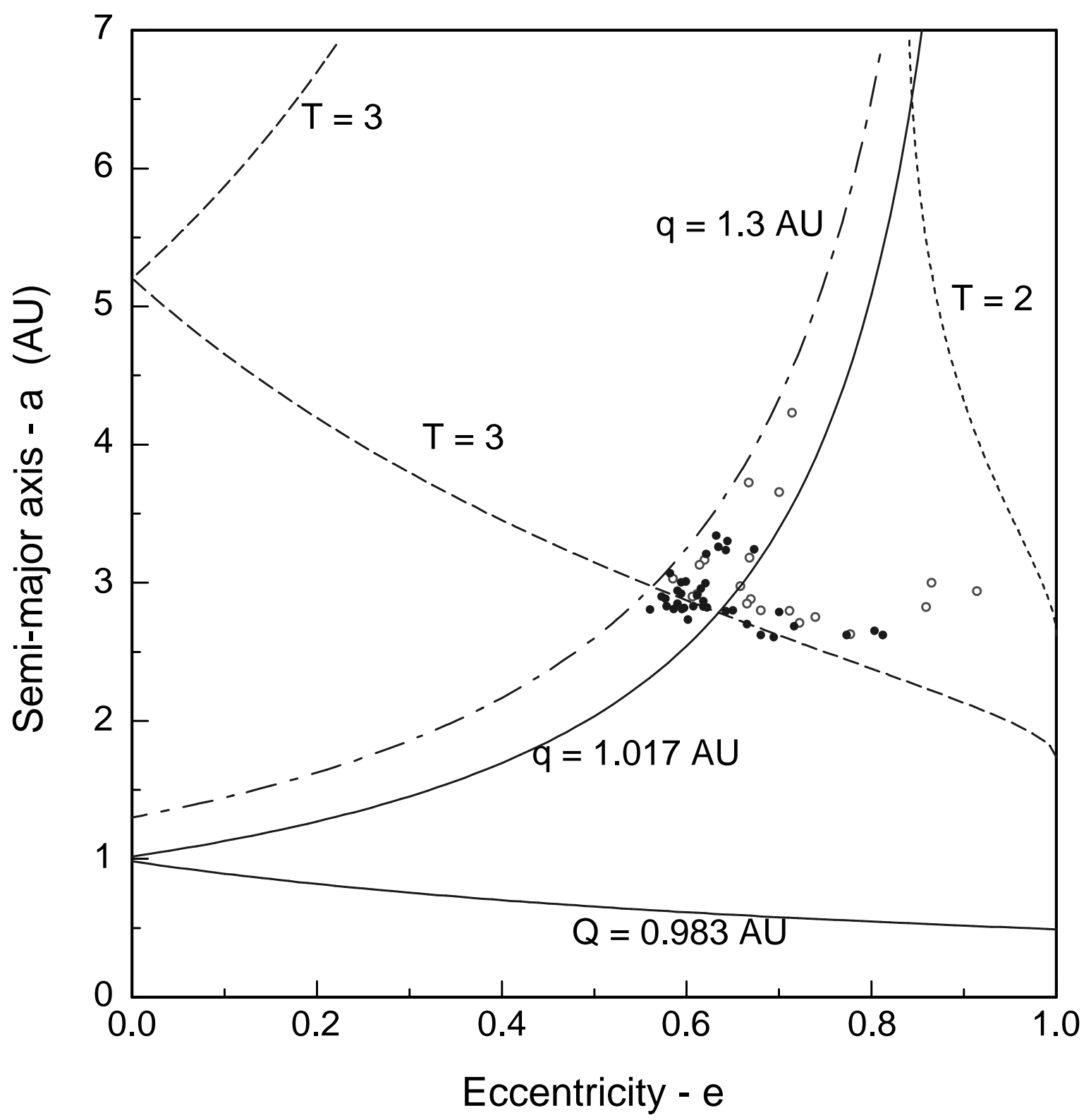

Figure 5. 


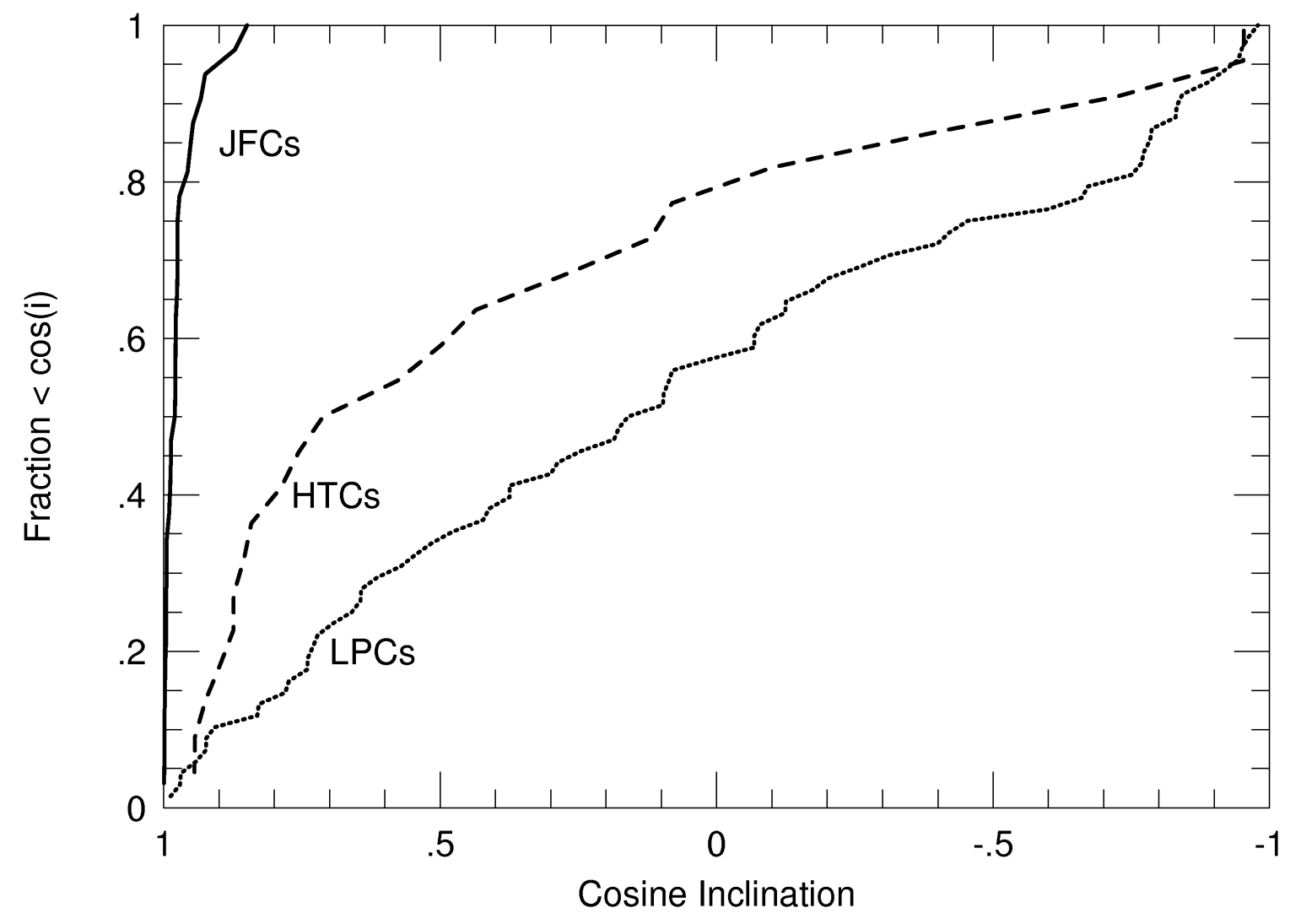

Figure 6. 